IFUP - TH $72 / 94$

UCB-PTH-94/29

hep-ph/9501334

LBL 36381

January 1995

\title{
Violations of lepton flavour and CP in supersymmetric unified theories
}

\author{
Riccardo Barbieri ${ }^{\dagger}$, Lawrence Hall ${ }^{\ddagger}$ and Alessandro Strumia ${ }^{\dagger}$ \\ $\dagger$ Dipartimento di Fisica, Università di Pisa \\ and INFN, Sezione di Pisa, I-56126 Pisa, Italy \\ $\ddagger$ Department of Physics, University of California at Berkeley, California 94720
}

\begin{abstract}
As a consequence of the large top quark Yukawa coupling, supersymmetric unified theories with soft supersymmetry breaking terms generated at the Planck scale predict lepton flavour and $\mathrm{CP}$ violating processes with significant rates.

The flavour violating parameters of the low energy theory are derived in both $\mathrm{SU}(5)$ and $\mathrm{SO}(10)$ theories, and are used to calculate the rate for $\mu \rightarrow e \gamma$. The sensitivity of the search for $\mu \rightarrow e \gamma$ is compared with that for $\mu \rightarrow e$ conversion in atoms, $\tau \rightarrow \mu \gamma$ and the electric dipole moment of the electron. The experimental search for these processes is shown to provide a very significant test of supersymmetric unification, especially in $\mathrm{SO}(10)$ but also in $\mathrm{SU}(5)$.
\end{abstract}

\footnotetext{
${ }^{1}$ Supported in part by U. S. Department of Energy under Contract DE-AC03-76SF00098 and in part by the National Science Foundation under grant PHY-90-21139.
} 


\section{Introduction}

The importance of looking for direct tests of unified theories cannot be overestated. As is well known, such an opportunity is essentially restricted to the study of violations of those conservation laws which are valid in the Standard Model as a consequence of exact "accidental" global symmetries. We refer to baryon number, $B$, and to the individual lepton numbers, $L_{e}, L_{\mu}$ and $L_{\tau}$.

In these respects, the violation of individual lepton numbers while preserving the overall lepton number, $L=L_{e}+L_{\mu}+L_{\tau}$, - hereafter called Lepton Flavour Violation (LFV) - plays a special role. If the Grand Unified Theory, characterized by a large mass scale $M_{\mathrm{G}}$, has the pure Standard Model as its low energy approximation, the rates for the corresponding LFV processes ( $\mu \rightarrow e \gamma, \mu \rightarrow e$ conversion, $\mu \rightarrow 3 e$, etc.) are unobservably small, since they are necessarily mediated by non-renormalizable effective interactions scaled by inverse powers of $M_{\mathrm{G}}$. On the contrary, in a supersymmetric unified theory with supersymmetry effectively broken at the Fermi scale, $m=\mathcal{O}\left(G_{\mathrm{F}}^{-1 / 2}\right)$, the rates for the LFV low energy processes are only suppressed by powers of $1 / m$ [1]. In general this would actually also be the case for $B$ and/or $L$-violating processes, like proton decay, strongly suggesting the need of matter parity (or $R$-parity) in a unified supersymmetric theory. Correspondingly, the LFV processes, consistent with matter parity unlike $B$ and/or $L$ violations, emerge as very interesting possible experimental signals of supersymmetric unification.

In a previous paper [2], two of us (R.B. and L.H.) have pointed out that the large Yukawa coupling of the top quark at the unification scale, $\lambda_{t \mathrm{G}}$, is an important source of flavour violation which reflects itself, via the unified couplings, in relatively large rates for general LFV processes. There it has been argued that the study of the corresponding experimental signals provides a test of supersymmetric unification at least as significant as the one that can be obtained from either proton decay or neutrino masses. In the present work we substantiate further this statement, by making an analytic study of the rates for one of the processes discussed in ref. [2], $\mu \rightarrow e \gamma$, in the full parameter space of the unified theory, both in $\mathrm{SU}(5)$ [3] and in $\mathrm{SO}(10)$ 四 and by subsequently comparing it with the other processes and quantities of interest. In addition to generating lepton flavour violating interactions, the large top quark Yukawa coupling also leads to important contributions to the electric dipole moment of the electron and neutron in $\mathrm{SO}(10)$ theories. In this paper we give the electric dipole moment over the full parameter space of the $\mathrm{SO}(10)$ unified theory.

The paper is organized as follows. In section 2 we discuss the physical mechanism which allows the top quark Yukawa coupling to generate large amplitudes for processes which violate individual lepton numbers. In section 3 we summarize the present information on $\lambda_{t \mathrm{G}}$ and we describe an upper bound on $\lambda_{t \mathrm{G}}$ arising from the infrared fixed point behaviour of the top Yukawa coupling above the unification scale up to $M_{\mathrm{Pl}}$. In section 4 we study the scaling of the supersymmetry breaking parameters in $\mathrm{SU}(5)$ with emphasis on the flavour violating effects due to $\lambda_{t \mathrm{G}}$. In section 5 we give, in $\mathrm{SU}(5)$, the pieces of the low energy Lagrangian relevant to the calculation of the LFV processes in the physical lepton and slepton basis. In section 6 we calculate the rate for $\mu \rightarrow e \gamma$ in the full space of parameters. In sections 7-9 we extend the analysis of sections 4-6 to the $\mathrm{SO}(10)$ case. In section 10 we discuss the $\mu \rightarrow e$ conversion in atoms and we establish the relative merit of the study of this process with respect to $\mu \rightarrow e \gamma$ in the search for a signal of lepton flavour violation. The same is done in section 11 for the $\tau \rightarrow \mu \gamma$ decay. Finally, in section 12, we study the relation between $\mu \rightarrow e \gamma$ and the electric dipole moment of the electron [5]. Our conclusions are drawn in section 13. Appendices A and B contain the analytic solutions of all the relevant Renormalization Group Equations from $M_{\mathrm{Pl}}$ to $M_{\mathrm{G}}$ (appendix A) and from $M_{\mathrm{G}}$ to $M_{Z}$ (appendix B), both in $\mathrm{SU}(5)$ and in $\mathrm{SO}(10)$.

\section{The origin of lepton flavour violation}

In this paper we study grand unified theories which incorporate weak-scale supersymmetry 6 and have the origin of supersymmetry breaking near at the Planck scale [7]. These theories lead to the successful weak mixing angle prediction, and, as a promising direction for unifying both the forces and the fundamental fermions, are currently receiving much attention. In all such theories, we find that the large top quark Yukawa coupling leads to a rate for $\mu \rightarrow e \gamma$ which can be reliably computed in terms of weak-scale parameters [2]. Over much of the interesting parameter space, the rate is within two orders of magnitude of the present experimental limit. At first sight, it is surprising that the top quark Yukawa coupling should lead to any violation of $L_{e}$ or $L_{\mu}$. What is the physical origin of this effect, and why is it not suppressed by inverse powers of $M_{\mathrm{G}}$ ? The answer lies in new flavour mixing matrices, which are analogous to the Cabibbo-Kobayashi-Maskawa matrix. 
In the standard model the quark mass eigenstate basis is reached by making independent rotations on the left-handed up and down type quarks, $u_{L}$ and $d_{L}$. However, these states are unified into a doublet of the weak $\mathrm{SU}(2)$ gauge group: $Q=\left(u_{L}, d_{L}\right)$. A relative rotation between $u_{L}$ and $d_{L}$ therefore leads to flavour mixing at the charged $W$ gauge vertex. This is the well-known Cabibbo-Kobayashi-Maskawa mixing. With massless neutrinos, the standard model has no analogous flavour mixing amongst the leptons: the charged lepton mass eigenstate basis can be reached by a rotation of the entire lepton doublet $L=\left(\nu_{L}, e_{L}\right)$.

How are these considerations of flavour mixing altered in supersymmetric unified theories? There are two new crucial ingredients. The first is provided by weak-scale supersymmetry, which implies that the quarks and leptons have scalar partners. The mass eigenstate basis for these squarks and sleptons requires additional flavour rotations. As an example, consider softly broken supersymmetric QED with three generations of charged leptons. There are three arbitrary mass matrices, one for the charged leptons, $e$, and one each for the left-handed and right-handed sleptons, $\tilde{e}_{L}$ and $\tilde{e}_{R}$. To reach the mass basis therefore requires relative rotations between $e_{L}$ and $\tilde{e}_{L}$ as well as between $e_{R}$ and $\tilde{e}_{R}$, resulting in two flavour mixing matrices at the photino gauge vertex.

In supersymmetric extensions of the standard model, these additional flavour-changing effects are known to be problematic. With a mixing angle comparable to the Cabibbo angle, a branching ratio for $\mu \rightarrow e \gamma$ of order $10^{-4}$ results. In the majority of supersymmetric models which have been constructed, such flavourchanging effects have been suppressed by assuming that the origin of supersymmetry breaking is flavour blind. In this case the slepton mass matrix is proportional to the unit matrix. The lepton mass matrix can then be diagonalized by identical rotations on $e_{L}$ and $\tilde{e}_{L}$ as well as on $e_{R}$ and $\tilde{e}_{R}$, without introducing flavour violating mixing matrices at the gaugino vertices. Slepton degeneracy renders lepton flavour mixing matrices non-physical.

The unification of quarks and leptons into larger multiplets provides the second crucial new feature in the origin of flavour mixing [1]. The weak unification of $u_{L}$ and $d_{L}$ into $Q$ is extended in $\mathrm{SU}(5)$ to the unification of $Q$ with $u_{L}^{c}$ and $e_{L}^{c}$ into a 10 dimensional multiplet $T\left(Q, u_{L}^{c}, e_{L}^{c}\right)$. Since higher unification leads to fewer multiplets, there are fewer rotations which can be made without generating flavour mixing matrices.

In any supersymmetric unified model there must be at least two coupling matrices, $\boldsymbol{\lambda}_{1}$ and $\boldsymbol{\lambda}_{2}$, which describe quark masses. If there is only one such matrix, it can always be diagonalized without introducing quark mixing. One of these coupling matrices, which we take to be $\boldsymbol{\lambda}_{1}$, must contain the large coupling, $\lambda_{t}$, which is responsible for the top quark mass. We choose to work in a basis in which $\boldsymbol{\lambda}_{1}$ is diagonal. The particles which interact via $\lambda_{t}$ are those which lie in the same unified multiplet with the top. In all unified models this includes a right-handed charged lepton, which we call $e_{L_{3}}^{c}$. This cannot be identified as the mass eigenstate $\tau_{L}^{c}$, because significant contributions to the charged lepton masses must come from the matrix $\boldsymbol{\lambda}_{2}$, which is not diagonal.

The assumption that the supersymmetry breaking mechanism is flavour blind leads to mass matrices for both $\tilde{e}_{L}$ and $\tilde{e}_{R}$ which are proportional to the unit matrix at the Planck scale, $M_{\mathrm{Pl}}$. As we have seen, without unified interactions, lepton superfield rotations can diagonalize the lepton mass matrix without introducing flavour mixing matrices. However, the unification prevents such rotations: the leptons are in the same multiplets as quarks, and the basis has already been chosen to diagonalize $\boldsymbol{\lambda}_{1}$. As the theory is renormalization group scaled to lower energies, the $\lambda_{t}$ interaction induces radiative corrections which suppress the mass of $\tilde{e}_{R_{3}}$ beneath that of $\tilde{e}_{R_{2}}$ and $\tilde{e}_{R_{1}}$. Beneath $M_{\mathrm{G}}$ the superheavy particles of the theory can be decoupled, leaving only the interactions of the minimal supersymmetric standard model. Now that the unified symmetry which relates quarks to leptons is broken, a lepton mass basis can be chosen by rotating lepton fields relative to quark fields. However, at these lower energies the sleptons are no longer degenerate, so that these rotations do induce lepton flavour mixing angles. Radiative corrections induced by $\lambda_{t}$ lead to slepton non-degeneracies, which render the lepton mixing angles physical [2].

This discussion provides the essence of the physics mechanism for lepton flavour violation in superunified models. Since the flavour mixing matrices have complex entries, they also lead to CP violation. It shows the effect to be generic to the idea of quark-lepton unification, requiring only that the superpartners have masses around the Fermi scale, and that supersymmetry breaking be present at the Planck scale. The imprint of the unified interactions is made on the soft supersymmetry breaking coefficients, including the scalar trilinears, which are taken to be flavour blind at the Planck scale. Eventually this imprint will be seen directly by studying the superpartner spectrum, but it can also be probed now by searching for $L_{e}$, $L_{\mu}, L_{\tau}$ and $\mathrm{CP}$ violating effects. 


\section{The top Yukawa coupling at the GUT scale}

The top Yukawa coupling at the unification scale, $\lambda_{t \mathrm{G}}$, plays a crucial role in the determination of the LFV effects discussed in this paper. In this section we therefore summarize the present information on $\lambda_{t \mathrm{G}}$ which comes from two different sources: the direct measurement of the top mass and, indirectly, the bottom/tau mass ratio.

The top Yukawa coupling $\lambda_{t \mathrm{G}}$ can of course be easily scaled down to determine its value at the weak scale $\lambda_{t}$ (see eq. (54b) of app. B). In turn, $\lambda_{t}$ determines the top quark pole mass via [8]

$$
M_{t}=\lambda_{t} v \sin \beta\left(1+\frac{4}{3} \frac{\alpha_{3}\left(M_{t}\right)}{\pi}+11.4 \frac{\alpha_{3}^{2}}{\pi^{2}}\right), \quad v=174 \mathrm{GeV},
$$

where, as usual, $\tan \beta=v_{\mathrm{u}} / v_{\mathrm{d}}$ is the ratio of the two light Higgs vacuum expectation values. Figure 11 shows $\lambda_{t \mathrm{G}}$ as function of the strong coupling constant $\alpha_{3}\left(M_{Z}\right)$, for $M_{t}=174 \pm 16 \mathrm{GeV}$ and for moderate $(\tan \beta=2)$ or relatively high values $(\tan \beta=10)$ of the ratio $v_{\mathrm{u}} / v_{\mathrm{d}}$. The rapid saturation for $\operatorname{large} \tan \beta$ implies that the lowest curve in fig. 1 is actually a lower bound on $\lambda_{t \mathrm{G}}$ for $m_{t}>158 \mathrm{GeV}$.

As is well known, the Yukawa superpotential of minimal SU(5) allows a prediction for the ratio $m_{b} / m_{\tau}$ as a function of $\lambda_{t \mathrm{G}}$ and $\alpha_{3}\left(M_{Z}\right)$ [9, 10]. This prediction is given for the running $b$-mass $m_{b}\left(m_{b}\right)$ in fig. 2, and is compared with the preferred value as determined from $\Upsilon$-physics. The dependence on $\tan \beta$ drops out in the ratio $m_{b} / m_{\tau}$, except for $\tan \beta \approx m_{t} / m_{b}$. For moderate values of $\tan \beta$ there is a clear consistency between fig. 1 and fig. 2 with a strong indication for a high value of $\lambda_{t \mathrm{G}}$. The consistency is weaker for larger $\tan \beta$, unless the top mass is close to $200 \mathrm{GeV}$, in the upper range of preliminary values indicated by the $\mathrm{CDF}$ experiment [11. In this case, of course, a rather high value of $\lambda_{t \mathrm{G}}$ is also indicated, resulting in a large flavour violation of the soft supersymmetry breaking parameters at the unification scale. For very large $\tan \beta$, close to $m_{t} / m_{b}$, consistency with $m_{b} / m_{\tau}$ is possible even for the smallest values of $\lambda_{t \mathrm{G}}$ allowed by the top mass. We have not studied this case in this paper. However, because the $\mu \rightarrow e \gamma$ amplitude always contains a term proportional to $\tan \beta$, the rate is always significant for such large values of $\tan \beta$.

For later purposes, it will be useful to know the behaviour of $\lambda_{t}$ at energies above $M_{\mathrm{G}}$, as determined from the Renormalization Group Equations (RGE). Assuming that the unified gauge coupling $g_{5}$ and $\lambda_{t}$ itself are the only relevant couplings, the RGEs are solved in appendix A at the one loop level. The solution for $\lambda_{t}$ displays an infrared fixed point. If $\lambda_{t}\left(M_{\mathrm{Pl}}\right)$ is large, but still perturbative, it will be drawn to the infrared fixed point at $M_{\mathrm{G}}$. The value of the coupling at the fixed point at $M_{\mathrm{G}}$ is larger for $\mathrm{SU}(5)$ than for $\mathrm{SO}(10)$, and depends on the one loop coefficient, $b_{\mathrm{G}}$, of the gauge $\beta$-function, as shown in figure 3 . The quantity $\lambda_{t \mathrm{G}}^{\max }$ plotted in this figure is the value of $\lambda_{t \mathrm{G}}$ for which the one loop evolved value of $\lambda_{t}\left(M_{\mathrm{Pl}}\right)$ becomes infinite. For all the numerical work of this paper we take $\lambda_{t \mathrm{G}}<\lambda_{t \mathrm{G}}^{\max }$, so that perturbation theory can be trusted. For larger values of $\lambda_{t \mathrm{G}}$ the theory becomes non-perturbative at scales beneath $M_{\mathrm{Pl}}$. Although we are unable to make computations for this case, the non-perturbative coupling is expected to generate large non-degeneracies amongst the scalars, leading to large rates for $\mu \rightarrow e \gamma$.

\section{Scaling of supersymmetry breaking parameters in SU(5)}

The messengers of flavour violation in the lepton sector are the soft supersymmetry breaking terms, which are therefore crucial to determine. Without having to specify the actual mechanism of supersymmetry breaking, nor the sector in which it takes place, we assume that it is transmitted to standard matter by supergravity couplings [7] and that it results, at the Planck scale, in universal soft breaking terms.

Standard matter occurs in the usual triplication of $10(T) \oplus \overline{5}(\bar{F})$ representations of SU(5), which are coupled to a $5(H)$ and a $\overline{5}(\bar{H})$ representation of Higgs supermultiplets in the Yukawa superpotential

$$
W=T_{i} \lambda_{i j}^{\mathrm{u}} T_{j} H+T_{i} \lambda_{i j}^{\mathrm{d}} \bar{F}_{j} \bar{H} \equiv T^{T} \boldsymbol{\lambda}^{\mathrm{u}} T H+T^{T} \boldsymbol{\lambda}^{\mathrm{d}} \bar{F} \bar{H}
$$

which we assume to be valid from $M_{\mathrm{G}}$ to the Planck scale. The full superpotential will contain other supermultiplets $\Sigma$, needed to break SU(5) but not directly coupled to matter. Assuming no large Yukawa couplings of the $\Sigma$ fields to the $H, \bar{H}$ multiplets, the $\Sigma$ fields affect the determination of the soft supersymmetry breaking terms at the GUT scale only via their contribution to the gauge $\beta$-function from $M_{\mathrm{G}}$ to $M_{\mathrm{Pl}}$. Unless otherwise specified, we shall take the $\mathrm{SU}(5) \beta$-function coefficient of the minimal Dimopoulos-Georgi model [6]. Different $\beta$-function coefficients mostly affect the rates for the LFV processes only through the restrictions that they induce on the range of the low energy parameters (see appendix A). 
Figure 1: The top Yukawa coupling at $M_{\mathrm{G}}$ for $\tan \beta=2$ (full lines) and $\tan \beta=10$ (dashed lines) for $M_{t}=158,174,192 \mathrm{GeV}$ (in increasing order), as function of $\alpha_{3}\left(M_{Z}\right)$.

Figure 2: The running $b$-quark mass in the $\alpha_{3}\left(M_{Z}\right), \lambda_{t \mathrm{G}}$ plane from $b / \tau$ unification. The darker area corresponds to $m_{b}\left(m_{b}\right)=4.25 \pm 0.10 \mathrm{GeV}$, as obtained from $\Upsilon$-physics.

Figure 3: Fixed point upper bounds on $\lambda_{t \mathrm{G}}$ in $\mathrm{SU}(5)$ and $\mathrm{SO}(10)$, as defined in the text, as functions of the one loop coefficient $b_{\mathrm{G}}$ of the gauge $\beta$-function.

The relevant part of the soft supersymmetry breaking Lagrangian, before SU(5) breaking, has the form

$$
-\mathcal{L}_{\text {soft }}=V_{\text {soft }}=\tilde{T}^{\dagger} \boldsymbol{m}_{T}^{2} \tilde{T}+\tilde{\bar{F}}^{\dagger} \boldsymbol{m}_{\bar{F}}^{2} \tilde{\bar{F}}+m_{H}^{2}|H|^{2}+m_{\bar{H}}^{2}|\bar{H}|^{2}+\tilde{T}^{T} \boldsymbol{A}^{\mathrm{u}} \boldsymbol{\lambda}^{\mathrm{u}} \tilde{T} H+\tilde{T}^{T} \boldsymbol{A}^{\mathrm{d}} \boldsymbol{\lambda}^{\mathrm{d}} \tilde{\bar{F}} \bar{H}
$$

with, at the Planck scale,

$$
\begin{gathered}
\boldsymbol{m}_{T}^{2}=\boldsymbol{m}_{\bar{F}}^{2}=m_{0}^{2} \mathbf{1}, \\
m_{H}^{2}=m_{\bar{H}}^{2}=m_{0}^{2}, \\
\boldsymbol{A}^{\mathrm{u}}=\boldsymbol{A}^{\mathrm{d}}=A_{0} \mathbf{1} .
\end{gathered}
$$

The renormalization of the parameters in (3) down to the GUT scale is most easily done by working in the basis where the Yukawa matrix $\boldsymbol{\lambda}^{\mathrm{u}}$ has diagonal form (hereafter called the u-basis). By keeping in the RGE only the one loop effects due to the SU(5) gauge coupling and to the third entry of $\boldsymbol{\lambda}^{\mathrm{u}}, \lambda_{33}^{\mathrm{u}}=\lambda_{t}$, it is simple to rescale down to $M_{\mathrm{G}}$ the soft breaking parameters (see appendix $\mathrm{A}$ ). Flavour universality is of course no longer maintained. In fact, the mass term for the ten-plet and the $A$-terms acquire the form

$$
\begin{aligned}
\boldsymbol{m}_{T \mathrm{G}}^{2} & =\operatorname{diag}\left(m_{T \mathrm{G}}^{2}, m_{T \mathrm{G}}^{2}, m_{T \mathrm{G}}^{2}-I_{\mathrm{G}}\right) \equiv m_{T \mathrm{G}}^{2} \mathbf{1}-\boldsymbol{I}_{\mathrm{G}}, \\
\boldsymbol{A}_{\mathrm{G}}^{\mathrm{d}} & =\operatorname{diag}\left(A_{d \mathrm{G}}, A_{d \mathrm{G}}, A_{d \mathrm{G}}-\frac{1}{3} I_{\mathrm{G}}^{\prime}\right) \equiv A_{d \mathrm{G}} \mathbf{1}-\frac{1}{3} \boldsymbol{I}_{\mathrm{G}}^{\prime}, \\
\boldsymbol{A}_{\mathrm{G}}^{\mathrm{u}} & =\operatorname{diag}\left(A_{u \mathrm{G}}-\frac{1}{3} I_{\mathrm{G}}^{\prime}, A_{u \mathrm{G}}-\frac{1}{3} I_{\mathrm{G}}^{\prime}, A_{u \mathrm{G}}-I_{\mathrm{G}}^{\prime}\right) .
\end{aligned}
$$

At the same time, the mass matrix of the five-plets maintains the universal form

$$
\boldsymbol{m}_{\bar{F} \mathrm{G}}^{2}=m_{\bar{F} \mathrm{G}}^{2} \mathbf{1}
$$

and the Yukawa coupling matrix $\boldsymbol{\lambda}^{\mathrm{u}}$ remains diagonal,

$$
\lambda_{\mathrm{G}}^{\mathrm{u}}=\operatorname{diag}\left(\lambda_{u \mathrm{G}}, \lambda_{c \mathrm{G}}, \lambda_{t \mathrm{G}}\right),
$$

whereas $\boldsymbol{\lambda}^{\mathrm{d}}$ gets renormalized to $\boldsymbol{\lambda}_{\mathrm{G}}^{\mathrm{d}}$. The explicit expressions for $m_{T \mathrm{G}}^{2}, m_{\bar{F} \mathrm{G}}^{2}, A_{d \mathrm{G}}, A_{u \mathrm{G}}, I_{\mathrm{G}}$ and $I_{\mathrm{G}}^{\prime}$, as well as the renormalization of the Higgs mass parameters are given in appendix A. The flavour breaking parameters $I_{\mathrm{G}}$ and $I_{\mathrm{G}}^{\prime}$ have a crucial dependence on the top Yukawa coupling at $M_{\mathrm{G}}, \lambda_{t \mathrm{G}}$ (see section 5).

In this paper we take a universal boundary condition for the soft supersymmetry breaking parameters at the Planck scale. How do our results depend on this assumption? A well motivated relaxation of this assumption is to allow soft scalar masses to be the most general allowed by the gauge symmetry and by a symmetry which interchanges one generation with another. This would satisfy flavour changing phenomenology without forcing identical Higgs and matter scalar masses, and would also allow the scalars in $T$ to have masses different from those in $\bar{F}$. Although extra parameters must be introduced, this generalization will not affect our results in a crucial way. More important would be the addition of small flavour changing scalar masses at the Planck scale, since they would lead directly to the processes which we discuss in this paper. These contributions would simply add to those which we calculate here. While cancellations cannot be excluded, we believe they would have to be accidental. For example, the contributions from the Planck scale boundary condition would arise from string physics and would be independent of the value of $M_{\mathrm{G}}$. On the other hand, the contributions calculated in this paper do depend on $M_{\mathrm{G}}$.

\section{The low energy Lagrangian in SU(5)}

After SU(5) breaking, the scaling down to low energy of the various parameters results in the low energy Lagrangian, whose relevant pieces are summarized for ease of the reader. They are, to first order in the Yukawa couplings: 
i. The slepton mass matrix

$$
-\mathcal{L}_{m}^{\mathrm{sl}}=\tilde{L}^{\dagger} \boldsymbol{m}_{L}^{2} \tilde{L}+\tilde{e}_{R}^{\dagger} \boldsymbol{m}_{e}^{2} \tilde{e}_{R}+\tilde{e}_{R}^{T}\left(\boldsymbol{A}^{\mathrm{e}}+\mathbf{1} \mu \tan \beta\right) \boldsymbol{\lambda}^{\mathrm{e}} \tilde{e}_{L} v_{\mathrm{d}}+\text { h.c. }
$$

where $\tilde{L}, \tilde{e}_{R}$ are 3 -vectors containing the $\mathrm{SU}(2)$ doublet and singlet sleptons,

$$
\boldsymbol{m}_{L}^{2}=m_{L}^{2} \mathbf{1}, \quad \boldsymbol{m}_{e}^{2}=m_{e}^{2} \mathbf{1}-\boldsymbol{I}_{\mathrm{G}}, \quad \boldsymbol{A}^{\mathrm{e}}=A_{e} \mathbf{1}-\frac{1}{3} \boldsymbol{I}_{\mathrm{G}}^{\prime},
$$

$m_{L}^{2}, m_{e}^{2}$ and $A_{e}$ are given in appendix $\mathrm{B}$, and a term proportional to the $\mu$ parameter has been explicitly introduced;

ii. the Higgs mass terms

$$
-\mathcal{L}_{m}=\left(m_{\mathrm{u}}^{2}+\mu^{2}\right)\left|h_{\mathrm{u}}\right|^{2}+\left(m_{\mathrm{d}}^{2}+\mu^{2}\right)\left|h_{\mathrm{d}}\right|^{2}-m_{\mathrm{ud}}^{2}\left(h_{\mathrm{u}} h_{\mathrm{d}}+\text { h.c. }\right)
$$

with $m_{\mathrm{u}}^{2}, m_{\mathrm{d}}^{2}$ given in eq. (62) of appendix B.

iii. the quarks and lepton mass terms

$$
\mathcal{L}_{Y}=Q^{T} \boldsymbol{\lambda}_{Z}^{\mathrm{u}} u_{L}^{c} \cdot v_{\mathrm{u}}+Q^{T} \boldsymbol{\lambda}_{Z}^{\mathrm{d}} d_{L}^{c} \cdot v_{\mathrm{d}}+e_{L}^{c T} \boldsymbol{\lambda}_{Z}^{\mathrm{e}} L \cdot v_{\mathrm{d}}
$$

where, in the u-basis, $\boldsymbol{\lambda}_{Z}^{\mathrm{u}}$ has kept its diagonal form and the matrices $\boldsymbol{\lambda}^{\mathrm{d}}$ and $\boldsymbol{\lambda}^{\mathrm{e}}$, equal at $M_{\mathrm{G}}$, have been shifted by the different renormalization effects due to $\lambda_{t}$ and the gauge couplings.

The LFV parameter $I_{\mathrm{G}}$ is directly related to the splitting between the $\tilde{\tau}_{R}$ and the $\tilde{e}_{R}\left(\tilde{\mu}_{R}\right)$. The $\tilde{\tau}_{R}$-mass is shown in figures 4 for fixed values of $\lambda_{t \mathrm{G}}$ and of the $\tilde{e}_{R}$-mass, as function of $A_{e}$ and of the wino mass $M_{2}$ in its full range, as determined from $m_{\tilde{e}_{R}}$ itself. We take the value of $\lambda_{t \mathrm{G}}$ such that $\lambda_{t \mathrm{G}}^{2}=0.8\left(\lambda_{t \mathrm{G}}^{\max }\right)^{2}$. The lightness of $\tilde{\tau}_{R}$ is of course a main consequence of the present picture as far as the superpartner spectrum is concerned. Another interesting consequence is the strong upper bound on the gaugino mass for any given $m_{\tilde{e}_{R}}$, which results in particular in the lightest supersymmetric particle being always the lightest neutralino. The $\tilde{\tau}_{R}$-mass has a negligible dependence on $\tan \beta$ for $m_{\tilde{e}_{R}}^{2} \gg M_{Z}^{2}$. For values of $m_{\tilde{e}_{R}}$ higher than $300 \mathrm{GeV}, m_{\tilde{\tau}_{R}}$ and $M_{2}$ rescale in the same way as $m_{\tilde{e}_{R}}$ itself does.

By diagonalizing $\boldsymbol{\lambda}_{Z}^{\mathrm{d}}$ and $\boldsymbol{\lambda}_{Z}^{\mathrm{e}}$, we have

$$
\begin{aligned}
\boldsymbol{\lambda}_{Z}^{\mathrm{d}} v_{\mathrm{d}} & =\boldsymbol{V}^{*} \boldsymbol{M}^{\mathrm{d}} \boldsymbol{U}^{\dagger} \\
\boldsymbol{\lambda}_{Z}^{\mathrm{e}} v_{\mathrm{d}} & =\boldsymbol{V}^{\mathrm{e} *} \boldsymbol{M}^{\mathrm{e}} \boldsymbol{U}^{\mathrm{e} \dagger}
\end{aligned}
$$

where $\boldsymbol{M}^{\mathrm{d}}, \boldsymbol{M}^{\mathrm{e}}$ are the diagonal mass matrices for down quarks and charged leptons, $\boldsymbol{U}=\boldsymbol{U}^{\mathrm{e}}, \boldsymbol{V}$ is the usual Cabibbo-Kobayashi-Maskawa matrix and, as an effect of the top Yukawa coupling, the matrix elements of $\boldsymbol{V}^{\mathrm{e}}$ are related to those of $\boldsymbol{V}$ by [12]

$$
V_{i j}^{\mathrm{e}}=y V_{i j} \quad \text { for } i \neq j \text { and }(i \text { or } j)=3, \quad V_{i j}^{\mathrm{e}}=V_{i j} \quad \text { otherwise }
$$

and $y$ is defined in eq. (56) of app. B. We ignore for the time being the fact that one does not obtain in this way the correct relation between the masses of the light leptons and down quarks, which are also related to each other by an appropriate renormalization group rescaling.

It is convenient to work in a mass eigenstate basis for the charge leptons, which is simply obtained by the redefinitions (with primed indices suppressed after eq. (14))

$$
\boldsymbol{V}^{\mathrm{e} \dagger} e_{L}^{c}=e_{L}^{c}, \quad \boldsymbol{U}^{\mathrm{e} \dagger} L=L^{\prime} .
$$

In the gaugino couplings, the rotation on the charged lepton doublets can be compensated by the same rotation of the full supermultiplets, since the $\mathrm{SU}(2)$ doublet slepton mass matrix has kept its diagonal form through renormalization, whereas this is not the case for the singlets $\tilde{e}_{R}$. As a consequence, the matrix $\boldsymbol{V}^{\mathrm{e}}$ appears in the neutralino couplings

$$
\mathcal{L}_{g}=\sqrt{2} g^{\prime} \sum_{n=1}^{4}\left[-\frac{1}{2} \overline{e_{L}} \tilde{e}_{L} N_{n}\left(H_{n \tilde{B}}+\cot \theta_{\mathrm{W}} H_{n \tilde{W}_{3}}\right)+\overline{\bar{e}_{L}^{c}} \boldsymbol{V}^{\mathrm{e} \dagger} \tilde{e}_{R} N_{n} H_{n \tilde{B}}+\text { h.c. }\right]
$$


Figure 4: Isoplots of $m_{\tilde{\tau}_{R}}$ in the $M_{2}, A_{e} / m_{\tilde{e}_{R}}$ plane for (a) $\lambda_{t \mathrm{G}}=1.4, m_{\tilde{e}_{R}}=100 \mathrm{GeV}, \tan \beta=2$ in SU(5), (b) $\lambda_{t \mathrm{G}}=1.4, m_{\tilde{e}_{R}}=100 \mathrm{GeV}, \tan \beta=10$ in $\mathrm{SU}(5)$, (c) $\lambda_{t \mathrm{G}}=1.4, m_{\tilde{e}_{R}}=300 \mathrm{GeV}, \tan \beta=2,10$ in $\mathrm{SU}(5)$, or, for $M_{2}<270 \mathrm{GeV}, \lambda_{t \mathrm{G}}=1.25, m_{\tilde{e}_{R}}=300 \mathrm{GeV}, \tan \beta=2,10$ in $\mathrm{SO}(10)$, (d) $\lambda_{t \mathrm{G}}=0.86$, $m_{\tilde{e}_{R}}=300 \mathrm{GeV}, \tan \beta=2,10$ in $\mathrm{SO}(10)$. In fig.s $4 \mathrm{a}$ a,b the isolines are separated by $10 \mathrm{GeV}$, in fig.s $4 \mathrm{c}, \mathrm{d}$ by $30 \mathrm{GeV}$.

Figure 5: Lepton flavour violating couplings in SU(5).

Figure 6: Diagrams giving rise to the decay $\mu \rightarrow e \gamma$ in $\mathrm{SU}(5)$. In figures $6 \mathrm{~b}, \mathrm{c}$, an external photon line is left understood, which can be attached to either of the scalar lines.

Figure 7: Isoplots of B.R. $(\mu \rightarrow e \gamma)$ in SU(5) in the $M_{2}, A_{e} / m_{\tilde{e}_{R}}$ plane for $\lambda_{t \mathrm{G}}=1.4, m_{\tilde{e}_{R}}=100 \mathrm{GeV}$ and (a) $\tan \beta=2, \mu<0$, (b) $\tan \beta=2, \mu>0$, (c) $\tan \beta=10, \mu<0$, (d) $\tan \beta=10, \mu>0$. The dashed (dotted) lines delimit regions where $m_{\tilde{\tau}_{R}}^{2}<0\left(\mu^{2}<0\right)$. The shaded area also extends to $m_{\tilde{\tau}_{R}}<45 \mathrm{GeV}$. The darker area shows a region where the rate is small, and passes through zero, due to a cancellation of terms. The dot-dashed line corresponds to the present experimental limit. For the CKM matrix elements we take $\left|V_{c b}\right|=0.04$ and $\left|V_{t d}\right|=0.01$.

Figure 8: Same as in fig. 团 for $m_{\tilde{e}_{R}}=300 \mathrm{GeV}$.

where $N_{n}$ are the four neutralino mass eigenstates, of mass $M_{n}$, related to the bino and the neutral wino by

$$
\begin{aligned}
\tilde{B} & =\sum_{n=1}^{4} N_{n} H_{n \tilde{B}}, \\
\tilde{W}_{3} & =\sum_{n=1}^{4} N_{n} H_{n \tilde{W}_{3}} .
\end{aligned}
$$

Notice that, in the slepton basis in which we are working, also the third term in the right side of (8) has non diagonal form, being

$$
-\mathcal{L}_{m}^{\text {n.d. }}=\left(A_{e}+\mu \tan \beta\right) \tilde{e}_{R}^{T} \boldsymbol{V}^{\mathrm{e} *} \boldsymbol{M}^{\mathrm{e}} \tilde{e}_{L}-\tilde{e}_{R}^{T} \frac{1}{3} \boldsymbol{I}_{\mathrm{G}}^{\prime} \boldsymbol{V}^{\mathrm{e} *} \boldsymbol{M}^{\mathrm{e}} \tilde{e}_{L}+\text { h.c. }
$$

\section{$6 \quad \mu \rightarrow e \gamma$ in supersymmetric $\mathbf{S U}(5)$}

The LFV couplings are summarized in fig. 5 . Correspondingly, if we neglect the electron mass and we work to first order in $m_{e} / m_{\mu}$ or $m_{\mu} / m_{\tau}$, the diagrams giving rise to the decay $\mu \rightarrow e \gamma$ are shown in fig. 6. Taking into account that the selectron, $\tilde{e}_{R}$, and smuon, $\tilde{\mu}_{R}$, singlets are degenerate at a common squared mass $m_{\tilde{e}_{R}}^{2}$, whereas they are split from the stau singlet $\tilde{\tau}_{R}$, of squared mass $m_{\tilde{\tau}_{R}}^{2}=m_{\tilde{e}_{R}}^{2}-I_{\mathrm{G}}$, and using the unitarity of the matrix $\boldsymbol{V}^{\mathrm{e}}$, one obtains the following contributions to the $\mu \rightarrow e \gamma$ decay amplitude

$$
\begin{aligned}
& \mathcal{A}_{\mu}(\mu \rightarrow e \gamma)=-i e \cdot \bar{u}_{e} i \sigma_{\mu \nu} q^{\nu} \frac{1-\gamma_{5}}{2} u_{\mu} F_{2} \\
& F_{2}^{(\mathrm{a})}=\frac{\alpha}{4 \pi \cos ^{2} \theta_{\mathrm{W}}} m_{\mu} V_{\tau \mu}^{\mathrm{e} *} V_{\tau e}^{\mathrm{e}}\left[G_{1}\left(m_{\tilde{\tau}_{R}}^{2}\right)-G_{1}\left(m_{\tilde{e}_{R}}^{2}\right)\right]
\end{aligned}
$$

b. from the diagram of figure $6 \mathrm{~b}$ :

$$
F_{2}^{(\mathrm{b})}=\frac{\alpha}{4 \pi \cos ^{2} \theta_{\mathrm{W}}} m_{\mu} V_{\tau \mu}^{\mathrm{e} *} V_{\tau e}^{\mathrm{e}}\left(A_{e}+\mu \tan \beta\right)\left[G_{2}\left(m_{\tilde{e}_{L}}^{2}, m_{\tilde{\tau}_{R}}^{2}\right)-G_{2}\left(m_{\tilde{e}_{L}}^{2}, m_{\tilde{e}_{R}}^{2}\right)\right]
$$

c. from the diagram of figure $6 \mathrm{c}$ :

$$
F_{2}^{(\mathrm{c})}=\frac{\alpha}{4 \pi \cos ^{2} \theta_{\mathrm{W}}} m_{\mu} V_{\tau \mu}^{\mathrm{e} *} V_{\tau e}^{\mathrm{e}}\left(-\frac{1}{3} I_{\mathrm{G}}^{\prime}\right) G_{2}\left(m_{\tilde{e}_{L}}^{2}, m_{\tilde{\tau}_{R}}^{2}\right)
$$

where

$$
G_{1}\left(m^{2}\right)=\sum_{n=1}^{4} \frac{H_{n \tilde{B}}^{2}}{M_{n}^{2}} g_{1}\left(\frac{m^{2}}{M_{n}^{2}}\right), \quad g_{1}(r)=\frac{-1}{6(r-1)^{4}}\left[2+3 r-6 r^{2}+r^{3}+6 r \ln r\right]
$$


and

$$
\begin{aligned}
G_{2}\left(m^{2}\right) & =\sum_{n=1}^{4} \frac{H_{n \tilde{B}}}{M_{n}}\left(H_{n \tilde{B}}+\cot \theta_{\mathrm{W}} H_{n \tilde{W}_{3}}\right) \cdot g_{2}\left(\frac{m^{2}}{M_{n}^{2}}\right), \\
G_{2}\left(m_{1}^{2}, m_{2}^{2}\right) & =\frac{G_{2}\left(m_{1}^{2}\right)-G_{2}\left(m_{2}^{2}\right)}{m_{1}^{2}-m_{2}^{2}}, \quad g_{2}(r)=\frac{1}{2(r-1)^{3}}\left[r^{2}-1-2 r \ln r\right] .
\end{aligned}
$$

Correspondingly, the decay rate is given by

$$
\Gamma(\mu \rightarrow e \gamma)=\frac{\alpha}{4} m_{\mu}^{3}\left|F_{2}\right|^{2}, \quad F_{2}=F_{2}^{(\mathrm{a})}+F_{2}^{(\mathrm{b})}+F_{2}^{(\mathrm{c})} .
$$

Equations $(19,20)$, together with the expressions of the parameters in the low energy Lagrangian as defined in the previous section and explicitly given in appendix B, allow the numerical calculation of the branching ratio B.R. $(\mu \rightarrow e \gamma)$, shown in fig. 7 and fig. 8 for $m_{\tilde{e}_{R}}$ equal to $100 \mathrm{GeV}$ and $300 \mathrm{GeV}$ respectively. For values of $m_{\tilde{e}_{R}}$ greater than $300 \mathrm{GeV}$ and fixed $A_{e} / m_{\tilde{e}_{R}}, M_{2} / m_{\tilde{e}_{R}}$ the branching ratio scale as $m_{\tilde{e}_{R}}^{-4}$. From (13), for the CKM matrix elements we take $\left|V_{c b}\right|=0.04$ and $\left|V_{t d}\right|=0.01$.

The set of independent parameters, on which the branching ratio depends, are $\left\{A_{0}, m_{0}^{2}, M_{5 \mathrm{Pl}}\right\}$ which determine the soft operators, the top quark Yukawa coupling, the coefficient of the one-loop gauge beta function of the unified theory, $b_{\mathrm{G}}$, the ratio of weak vacuum expectation values, $\tan \beta$, and the Higgs mixing parameter $\mu$. We choose to exchange $\left\{A_{0}, m_{0}^{2}, M_{5 \mathrm{Pl}}\right\}$ for the physically more interesting set $\left\{A_{e}, m_{\tilde{e}_{R}}^{2}, M_{2}\right\}$, where $A_{e}$ is the light generation lepton $A$-parameter, $m_{\tilde{e}_{R}}$ is the mass of the right-handed selectron and $M_{2}$ is the weak scale gaugino mass parameter for $\mathrm{SU}(2)$. In appendix A the full dependence of quantities on $b_{\mathrm{G}}$ is given, and is found to be mild, hence we have chosen the minimal value $b_{\mathrm{G}}=-3$. The $\mu$ parameter which enters in $F_{2}^{(\mathrm{b})}$, eq. (19b), and also in the neutralino mass matrix, is expressed, up to its sign, in terms of the other parameters by means of the electroweak symmetry breaking relation

$$
\mu^{2}=-\frac{M_{Z}^{2}}{2}-\frac{m_{\mathrm{d}}^{2}-m_{\mathrm{u}}^{2} \tan ^{2} \beta}{1-\tan ^{2} \beta}
$$

with $m_{\mathrm{u}}^{2}, m_{\mathrm{d}}^{2}$ given in appendix $\mathrm{B}$.

\section{Scaling of supersymmetry breaking parameters in $\mathrm{SO}(10)$}

In the case of $\mathrm{SO}(10)$ gauge symmetry, in fact as one of its most attractive features, the quarks and leptons of a single generation are the components of a single 16-dimensional spinorial representation $\Psi$. This is a crucial feature for the problem at hand; it causes all the scalars of the third generation, and not only those in the 10 of SU(5), to be lighter than the corresponding scalars in the first and the second generation. In turn, and at variance with SU(5), LFV interactions arise also involving the left handed sleptons. With this in mind, the considerations of the previous sections can be straightforwardly extended to the $\mathrm{SO}(10)$ case, after specifying the Yukawa superpotential and the gauge $\beta$-function, at one loop, at the Planck scale. For simplicity we assume that $\mathrm{SO}(10)$ is broken at once to the low energy standard group at $M_{\mathrm{G}}$.

In $\mathrm{SO}(10)$ gauge theories a single Yukawa interaction of the three spinorial matter multiplets $\Psi_{i}$ to a vector 10-dimensional Higgs representation $\Phi, \Psi^{T} \boldsymbol{\lambda} \Psi \Phi$, does not describe any intergenerational mixing, since $\Psi$ can be rotated to make $\boldsymbol{\lambda}$ diagonal. To describe the mixing, we introduce two 10-plets, $\Phi_{\mathrm{u}}$ and $\Phi_{\mathrm{d}}$, in the superpotential [5]

$$
W_{\mathrm{SO}(10)}=\Psi^{T} \boldsymbol{\lambda}^{\mathrm{u}} \Psi \Phi_{\mathrm{u}}+\Psi^{T} \boldsymbol{\lambda}^{\mathrm{d}} \Psi \Phi_{\mathrm{d}}
$$

and we assume that the light Higgs doublets $h_{\mathrm{u}}$ (with weak hypercharge $Y=+1 / 2$ ) and $h_{\mathrm{d}}$ (with weak hypercharge $Y=-1 / 2$ ) lie respectively in $\Phi_{\mathrm{u}}$ and $\Phi_{\mathrm{d}}$. As in the $\mathrm{SU}(5)$ case, this superpotential is taken to be valid already at the Planck scale. Furthermore, here too it is preferable to work in the basis where $\boldsymbol{\lambda}^{\mathrm{u}}$, which is responsible of the $Q=2 / 3$ quark masses, is diagonal. In analogy with equations (㺼), at the Planck scale we take

$$
\boldsymbol{m}_{\Psi}^{2}=m_{0}^{2} \mathbf{1}, \quad m_{\Phi_{\mathrm{u}}}^{2}=m_{\Phi_{\mathrm{d}}}^{2}=m_{0}^{2}, \quad \boldsymbol{A}^{\mathrm{u}}=\boldsymbol{A}^{\mathrm{d}}=A_{0} \mathbf{1}
$$

\footnotetext{
${ }^{2}$ In the limit of small $I_{\mathrm{G}}$, this rate agrees with the analytic expression given in ref. [2]. However, in view of the values actually taken by $I_{\mathrm{G}}$, the expansion generally gives a poor approximation to the correct rate. Previous calculations of the $\mu \rightarrow e \gamma$ rate for special values of the gaugino and slepton masses were made in references [13].
} 
After renormalization at the unification scale, we have

$$
-\mathcal{L}_{\text {soft }}=V_{\text {soft }}=\tilde{\Psi}^{\dagger} \boldsymbol{m}_{\Psi \mathrm{G}}^{2} \tilde{\Psi}+m_{\Phi_{\mathrm{u}}}^{2}\left|\Phi_{\mathrm{u}}\right|^{2}+m_{\Phi_{\mathrm{d}}}^{2}\left|\Phi_{\mathrm{d}}\right|^{2}+\tilde{\Psi}^{T} \boldsymbol{A}_{\mathrm{G}}^{\mathrm{u}} \boldsymbol{\lambda}_{\mathrm{G}}^{\mathrm{u}} \tilde{\Psi} \Phi_{\mathrm{u}}+\tilde{\Psi}^{T} \boldsymbol{A}_{\mathrm{G}}^{\mathrm{d}} \boldsymbol{\lambda}_{\mathrm{G}}^{\mathrm{d}} \tilde{\Psi} \Phi_{\mathrm{d}}
$$

where

$$
\begin{aligned}
\boldsymbol{m}_{\Psi \mathrm{G}}^{2} & =\operatorname{diag}\left(m_{\Psi \mathrm{G}}^{2}, m_{\Psi \mathrm{G}}^{2}, m_{\Psi \mathrm{G}}^{2}-I_{\mathrm{G}}\right) \equiv m_{\Psi \mathrm{G}}^{2} \mathbf{1}-\boldsymbol{I}_{\mathrm{G}}, \\
\boldsymbol{A}_{\mathrm{G}}^{\mathrm{d}} & =\operatorname{diag}\left(A_{d \mathrm{G}}, A_{d \mathrm{G}}, A_{d \mathrm{G}}-\frac{5}{7} I_{\mathrm{G}}^{\prime}\right) \equiv A_{d \mathrm{G}} \mathbf{1}-\frac{5}{7} \boldsymbol{I}_{\mathrm{G}}^{\prime}, \\
\boldsymbol{A}_{\mathrm{G}}^{\mathrm{u}} & =\operatorname{diag}\left(A_{u \mathrm{G}}-\frac{2}{7} I_{\mathrm{G}}^{\prime}, A_{u \mathrm{G}}-\frac{2}{7} I_{\mathrm{G}}^{\prime}, A_{u \mathrm{G}}-I_{\mathrm{G}}^{\prime}\right) .
\end{aligned}
$$

When possible we also keep the same notation as in the $\mathrm{SU}(5)$ case, but of course the relations of the various quantities, e.g. $I_{\mathrm{G}}$ in eq.s $(24 \mathrm{a})$ and $(5 \mathrm{a})$, to the input parameters at the Planck scale change. These relations in the $\mathrm{SO}(10)$ case are given in appendix $\mathrm{A}$, as function of the one loop coefficient of the gauge $\beta$-function. In the text we take $b_{\mathrm{G}}=-3$.

\section{The low energy lagrangian in $\mathrm{SO}(10)$}

The further scaling down from $M_{\mathrm{G}}$ to the weak scale of the different parameters gives rise to the low energy lagrangian with the same form as in eq. (8), except that now also the diagonal squared mass matrix of the left handed sleptons has a split third eigenvalue

$$
\boldsymbol{m}_{L \mathrm{G}}^{2}=m_{L \mathrm{G}}^{2} \mathbf{1}-\boldsymbol{I}_{\mathrm{G}} .
$$

The masses of the third generation sfermions are all reduced relative to the ones of the first two generations. For example the $\tilde{\tau}_{R}$-mass shows approximately the same pattern as in the $\mathrm{SU}(5)$ case for a correspondingly lower value of $\lambda_{t \mathrm{G}}$ by a relative amount $\lambda_{t \mathrm{G}}^{\max }(\mathrm{SO}(10)) / \lambda_{t \mathrm{G}}^{\max }(\mathrm{SU}(5)) \approx 0.87$ (see fig. 价.

On the other hand, in the fermion mass terms, the symmetry in flavour space of the $\mathrm{SO}(10)$ coupling $16_{i} 16_{j} 10$ gives rise to a symmetric lepton (or down) mass matrix, so that, in eq. (12), $\boldsymbol{U}^{\mathrm{e}}=\boldsymbol{V}^{\mathrm{e}}$.

As before, to calculate the amplitudes for the LFV processes, it is convenient to go to the mass-eigenstate basis for the charged leptons. At variance with the $\mathrm{SU}(5)$ case, however, this time the term $\boldsymbol{I}_{\mathrm{G}}$ in (25) prevents a counter-rotation also in the left-handed sleptons. As a consequence the flavour changing matrix $\boldsymbol{V}^{\mathrm{e}}$ appears in all the gaugino couplings, which acquire the form (for all terms involving the charged leptons)

$$
\begin{aligned}
\mathcal{L}_{g}=\sqrt{2} g^{\prime} \sum_{n=1}^{4}\left[-\frac{1}{2} \overline{e_{L}} \boldsymbol{V}^{\mathrm{e} \dagger} \tilde{e}_{L} N_{n}\left(H_{n \tilde{B}}+\cot \theta_{\mathrm{W}} H_{n \tilde{W}_{3}}\right)+\overline{e_{L}^{c}} \boldsymbol{V}^{\mathrm{e}} \tilde{e}_{R} N_{n} H_{n \tilde{B}}+\text { h.c. }\right] \\
+g \sum_{c=1}^{2}\left[\overline{e_{L}} \boldsymbol{V}^{\mathrm{e} \dagger} \tilde{\nu}_{L}\left(\chi_{c} K_{c \tilde{W}}\right)+\text { h.c. }\right]
\end{aligned}
$$

where $\chi_{c}$ are the two chargino mass eigenstates, related to the charged wino by

$$
\tilde{W}=\sum_{c=1}^{2} \chi_{c} K_{c \tilde{W}}
$$

and $\tilde{\nu}_{L}$ is the 3 -vector of the left-handed sneutrinos, which, apart from $\mathrm{SU}(2) \otimes \mathrm{U}(1)$ breaking, are degenerate with the charged left-handed sleptons.

Finally, as in SU(5), there is still a non diagonal 'chirality breaking' scalar mass term

$$
-\mathcal{L}_{m}^{\text {n.d. }}=\left(A_{e}+\mu \tan \beta\right) \tilde{e}_{R}^{T} \boldsymbol{V}^{\mathrm{e} *} \boldsymbol{M}^{\mathrm{e}} \boldsymbol{V}^{\mathrm{e} \dagger} \tilde{e}_{L}-\frac{1}{2} \tilde{e}_{R}^{T}\left\{\frac{5}{7} \boldsymbol{I}_{\mathrm{G}}^{\prime}, \boldsymbol{V}^{\mathrm{e} *} \boldsymbol{M}^{\mathrm{e}} \boldsymbol{V}^{\mathrm{e} \dagger}\right\} \tilde{e}_{L}+\text { h.c. }
$$

All the LFV couplings in the $\mathrm{SO}(10)$ case are summarized in fig. 9 .

\section{$9 \quad \mu \rightarrow e \gamma$ in supersymmetric $\mathbf{S O}(\mathbf{1 0})$}

The Feynman diagrams contributing to $\mu \rightarrow e \gamma$ in $\mathrm{SO}(10)$ are shown in figure 10 (for vanishing electron mass). The ones in figures $10 \mathrm{a}_{R}, \mathrm{a}_{L}, \mathrm{~d}$, with the helicity flip in the external fermion line, give an amplitude proportional to the muon mass, whereas the diagrams of figures $10 \mathrm{~b}_{L, R}, \mathrm{c}_{L, R}, \mathrm{c}_{L, R}^{\prime}$, with the helicity flip on the internal fermion line, have a dominant term proportional to the tau mass. As such, they dominate the decay rate in all of the physically allowed space of parameters. In the approximation of only keeping the 
Figure 9: Lepton flavour violating couplings in $\mathrm{SO}(10)$.

Figure 10: Diagrams giving rise to the decay $\mu \rightarrow e \gamma$ in $\mathrm{SO}(10)$. The graphs $10 \mathrm{a}_{L}, \mathrm{~b}_{L}, \mathrm{c}_{L}, \mathrm{c}_{L}^{\prime}$ involving an external right handed muon and an internal neutralino are not displayed. As in fig. $6 \mathrm{~b}, \mathrm{c}$, in fig. 10b,c, $\mathrm{c}^{\prime}$ an external photon line is understood.

Figure 11: Isoplots of B.R. $(\mu \rightarrow e \gamma)$ in $\mathrm{SO}(10)$ for $m_{\tilde{e}_{R}}=300 \mathrm{GeV}, \lambda_{t \mathrm{G}}=1.25$ and all other parameters as in fig. 7 .

Figure 12: Same as in fig. 11 for $\lambda_{t \mathrm{G}}=0.86$.

Figure 13: Isoplots of C.R. $\left(\mu \rightarrow e\right.$ in Ti) in $\mathrm{SU}(5)$ for $m_{\tilde{e}_{R}}=100$ or $300 \mathrm{GeV}, \lambda_{t \mathrm{G}}=1.4$ and $\tan \beta=2$.

Figure 14: Box diagrams contributing to $\mu \rightarrow e$ conversion in $\mathrm{SU}(5)$.

terms proportional to $m_{\tau}$, the left-handed and the right-handed muon have equal decay amplitudes, from the diagrams $10 \mathrm{~b}_{R}, \mathrm{c}_{R}, \mathrm{c}_{R}^{\prime}$ and $10 \mathrm{~b}_{L}, \mathrm{c}_{L}, \mathrm{c}_{L}^{\prime}$ respectively, which however do not interfere with each other for vanishing electron mass.

From the diagram of figure $10 \mathrm{~b}_{R}$ one has

$$
\begin{aligned}
F_{2}^{\left(\mathrm{b}_{R}\right)}= & \frac{\alpha}{4 \pi \cos ^{2} \theta_{\mathrm{W}}} m_{\tau} V_{\tau \mu}^{\mathrm{e}} V_{\tau e}^{\mathrm{e}}\left(V_{\tau \tau}^{\mathrm{e} *}\right)^{2}\left(A_{e}+\mu \tan \beta\right) \times \\
& \times\left[G_{2}\left(m_{\tilde{\tau}_{L}}^{2}, m_{\tilde{\tau}_{R}}^{2}\right)-G_{2}\left(m_{\tilde{e}_{L}}^{2}, m_{\tilde{\tau}_{R}}^{2}\right)-G_{2}\left(m_{\tilde{\tau}_{L}}^{2}, m_{\tilde{e}_{R}}^{2}\right)+G_{2}\left(m_{\tilde{e}_{L}}^{2}, m_{\tilde{e}_{R}}^{2}\right)\right],
\end{aligned}
$$

whereas, from the diagram of figure $10 c_{R}, c_{R}^{\prime}$ one has

$$
\begin{aligned}
F_{2}^{\left(\mathrm{c}_{R}\right)}+F_{2}^{\left(\mathrm{c}_{R}^{\prime}\right)}= & \frac{\alpha}{4 \pi \cos ^{2} \theta_{\mathrm{W}}} m_{\tau} V_{\tau \mu}^{\mathrm{e}} V_{\tau e}^{\mathrm{e}}\left(V_{\tau \tau}^{\mathrm{e} *}\right)^{2}\left(-\frac{5}{7} I_{\mathrm{G}}^{\prime}\right) \times \\
& \times\left[G_{2}\left(m_{\tilde{\tau}_{L}}^{2}, m_{\tilde{\tau}_{R}}^{2}\right)-\frac{1}{2} G_{2}\left(m_{\tilde{e}_{L}}^{2}, m_{\tilde{\tau}_{R}}^{2}\right)-\frac{1}{2} G_{2}\left(m_{\tilde{\tau}_{L}}^{2}, m_{\tilde{e}_{R}}^{2}\right)\right] .
\end{aligned}
$$

For the decay rate one has

$$
\Gamma(\mu \rightarrow e \gamma)=\frac{\alpha}{2} m_{\mu}^{3}\left|F_{2}\right|^{2}, \quad F_{2}=F_{2}^{\left(\mathrm{b}_{R}\right)}+F_{2}^{\left(\mathrm{c}_{R}\right)}+F_{2}^{\left(\mathrm{c}_{R}^{\prime}\right)} .
$$

The isoplots of B.R. $(\mu \rightarrow e \gamma)$ are shown in figures 11, 12 .

\section{$10 \mu \rightarrow e$ conversion}

Keeping only the vector coupling to the nucleus $\mathcal{N}$, the general amplitude for $\mu \rightarrow e$ conversion process can be written as

$$
\mathcal{A}=i e^{2}\left[\overline{\mathcal{N}} \gamma^{\mu} \mathcal{N}\right]\left[\bar{u}_{e}\left(g_{1 R} \gamma_{\mu} \mathcal{P}_{R}-g_{2 R} \frac{i \sigma_{\mu \nu} q^{\nu}}{m_{\mu}} \mathcal{P}_{L}\right) u_{\mu}\right]+(R \leftrightarrow L) .
$$

This amplitude gives rise to the coherent conversion rate

$$
\Gamma(\mu \rightarrow e)=4 \alpha^{5} \frac{Z_{\text {eff }}^{4}}{Z}|F(q)|^{2} m_{\mu}^{5}\left(\left|g_{1 R}-g_{2 R}\right|^{2}+\left|g_{1 L}-g_{2 L}\right|^{2}\right) .
$$

where $Z$ is the charge of the nucleus, $Z_{\text {eff }}$ is an effective charge and $F(q)$ the nuclear form factor 14.

In our case, the amplitude receives contributions both from penguin-type $(\mathrm{P})$ and from box $(\mathrm{B})$ diagrams. More precisely, to leading order in the lepton and light quark masses, the penguin diagrams contribute both to $g_{1}$ and $g_{2}$, unlike the box diagrams, which only contribute to $g_{1}$

$$
g_{1}=g_{1}^{\mathrm{B}}+g_{1}^{\mathrm{P}}, \quad g_{2}=g_{2}^{\mathrm{P}} .
$$

If we define, in analogy with eq. (18), the general off-shell $\mu \rightarrow e \gamma$ amplitude $\mathcal{A}_{\mu}(\mu \rightarrow e \gamma)$ as

$$
\mathcal{A}_{\mu}(\mu \rightarrow e \gamma)=-i e \cdot \bar{u}_{e}\left[q^{2} F_{1 R} \gamma_{\mu} \mathcal{P}_{R}+i \sigma_{\mu \nu} q^{\nu} F_{2 R} \mathcal{P}_{L}\right] u_{\mu}+(R \leftrightarrow L)
$$


one has

$$
\begin{aligned}
& g_{1 L, R}^{\mathrm{P}}=Z F_{1 L, R} . \\
& g_{2 L, R}^{\mathrm{P}}=Z F_{2 L, R} / m_{\mu} .
\end{aligned}
$$

In the case of the $\mathrm{SO}(10)$ gauge theory, only the magnetic penguin-type amplitudes $g_{2 L, R}^{\mathrm{P}}$ have a term proportional to $m_{\tau}$, as discussed in section 9. Furthermore $g_{2 L}^{\mathrm{P}}=g_{2 R}^{\mathrm{P}} \equiv g_{2}^{\mathrm{P}}$. Therefore, apart from terms of relative order $\left(m_{\mu} / m_{\tau}\right)^{2}$, in view of eq. $(36 \mathrm{~b})$,

$$
\Gamma(\mu \rightarrow e)=16 \alpha^{4} Z_{\mathrm{eff}}^{4} Z|F(q)|^{2} \Gamma(\mu \rightarrow e \gamma) .
$$

In the case of $\mathrm{Ti}_{22}^{48}$, for which [14 $Z=22, Z_{\text {eff }}=17.6,|F(q)|=0.54$, taking into account the experimental value for the capture rate $\Gamma(\mu$ capture in $\mathrm{Ti})=(2.590 \pm 0.012) \cdot 10^{6} / \mathrm{sec}$, we obtain

$$
\text { C.R. }(\mu \rightarrow e \text { in } \mathrm{Ti}) \equiv \frac{\Gamma(\mu \rightarrow e \text { in } \mathrm{Ti})}{\Gamma(\mu \text { capture in Ti })}=0.5 \cdot 10^{-2} \text { B.R. }(\mu \rightarrow e \gamma)
$$

or, normalizing both ratios to the present upper limits [16, 17]

$$
\frac{\text { C.R. }(\mu \rightarrow e \text { in } \mathrm{Ti})}{10^{-12}}=0.25 \frac{\text { B.R. }(\mu \rightarrow e \gamma)}{4.9 \cdot 10^{-11}} .
$$

Using relation (38), the contours of figures 11 and 12 can be relabelled with values of C.R. $(\mu \rightarrow e$ in Ti). This relation holds whenever the $g_{1}$ form factor contribution can be neglected. In the $\mathrm{SO}(10)$ model this is always the case, giving this relation a wide applicability; wider than the applicability of the results for the rates themselves. For example, this relation is independent of $\lambda_{t \mathrm{G}}$, the form and values of the supersymmetry breaking parameters and the form of the RGE above $M_{\mathrm{G}}$ (even if the theory becomes non-perturbative).

Contrary to the $\mathrm{SO}(10)$ case, in $\mathrm{SU}(5)$, all the form factors in eq. (32) contribute in principle at the same general level.

In practice, the contribution of the magnetic form factor $g_{2}^{\mathrm{P}}$ is almost always numerically dominant also in the $\mathrm{SU}(5)$ case, at least as long as the gaugino mass parameter $M_{2}$ is not close to zero or the magnetic form factor has no accidental cancellation, which may occur for $\mu<0$. Relative to the penguin contribution $g_{1}^{\mathrm{P}}$, this comes about because the electric form factor has no term proportional to $A_{e}$ and $\mu \tan \beta$. The box contribution has a quite different structure from the penguin contribution. From the diagrams of figure 14 one obtains for the effective Hamiltonian involving the quark fields

$$
\begin{aligned}
\mathcal{H}^{\mathrm{B}}(\mu \rightarrow e)= & -i \frac{\alpha^{2}}{\cos ^{4} \theta_{\mathrm{W}}} V_{e \tau}^{\mathrm{e}} V_{\mu \tau}^{\mathrm{e} *}\left[\overline{e_{R}} \gamma_{\mu} \mu_{R}\right] \times \\
& \times\left[\frac{1}{36}\left(c_{u_{L}} \cdot \overline{u_{L}} \gamma^{\mu} u_{L}+c_{d_{L}} \cdot \overline{d_{L}} \gamma^{\mu} d_{L}\right)-\frac{4}{9}\left(c_{u_{R}} \cdot \overline{u_{R}} \gamma^{\mu} u_{R}\right)-\frac{1}{9}\left(c_{d_{R}} \cdot \overline{d_{R}} \gamma^{\mu} d_{R}\right)\right]
\end{aligned}
$$

with

$$
\begin{aligned}
c_{u_{L}}= & \sum_{n, m=1}^{4}\left[B\left(m_{\tilde{\tau}_{R}}^{2}, m_{\tilde{u}_{L}}^{2}, M_{n}, M_{m}\right)-B\left(m_{\tilde{e}_{R}}^{2}, m_{\tilde{u}_{L}}^{2}, M_{n}, M_{m}\right)\right] \times \\
& \times H_{n \tilde{B}} H_{m \tilde{B}}\left(H_{n \tilde{B}}+3 H_{n \tilde{W}_{3}} \cot \theta_{\mathrm{W}}\right)\left(H_{m \tilde{B}}+3 H_{m \tilde{W}_{3}} \cot \theta_{\mathrm{W}}\right) \\
c_{d_{L}}= & \sum_{n, m=1}^{4}\left[B\left(m_{\tilde{\tau}_{R}}^{2}, m_{\tilde{d}_{L}}^{2}, M_{n}, M_{m}\right)-B\left(m_{\tilde{e}_{R}}^{2}, m_{\tilde{d}_{L}}^{2}, M_{n}, M_{m}\right)\right] \times \\
& \times H_{n \tilde{B}} H_{m \tilde{B}}\left(H_{n \tilde{B}}-3 H_{n \tilde{W}_{3}} \cot \theta_{\mathrm{W}}\right)\left(H_{m \tilde{B}}-3 H_{m \tilde{W}_{3}} \cot \theta_{\mathrm{W}}\right) \\
c_{u_{R}}= & \sum_{n, m=1}^{4}\left[B\left(m_{\tilde{\tau}_{R}}^{2}, m_{\tilde{u}_{R}}^{2}, M_{n}, M_{m}\right)-B\left(m_{\tilde{e}_{R}}^{2}, m_{\tilde{u}_{R}}^{2}, M_{n}, M_{m}\right)\right] H_{n \tilde{B}}^{2} H_{m \tilde{B}}^{2} \\
c_{d_{R}}= & \sum_{n, m=1}^{4}\left[B\left(m_{\tilde{\tau}_{R}}^{2}, m_{\tilde{d}_{R}}^{2}, M_{n}, M_{m}\right)-B\left(m_{\tilde{e}_{R}}^{2}, m_{\tilde{d}_{R}}^{2}, M_{n}, M_{m}\right)\right] H_{n \tilde{B}}^{2} H_{m \tilde{B}}^{2}
\end{aligned}
$$




$$
B\left(m_{1}^{2}, m_{2}^{2}, M_{1}, M_{2}\right) \equiv i(4 \pi)^{2} \int \frac{d^{4} k}{(2 \pi)^{4}} \frac{k^{2}+2 M_{1} M_{2}}{\left(k^{2}-m_{1}^{2}\right)\left(k^{2}-m_{2}^{2}\right)\left(k^{2}-M_{1}^{2}\right)\left(k^{2}-M_{2}^{2}\right)} .
$$

Consequently, by means of ( $N$ is the number of neutrons in the nucleus)

$$
\begin{aligned}
\left\langle\mathcal{N}\left|\overline{u_{L, R}} \gamma_{\mu} u_{L, R}\right| \mathcal{N}\right\rangle & \approx(Z+N / 2) \overline{\mathcal{N}} \gamma_{\mu} \mathcal{N} \\
\left\langle\mathcal{N}\left|\overline{d_{L, R}} \gamma_{\mu} d_{L, R}\right| \mathcal{N}\right\rangle & \approx(N+Z / 2) \overline{\mathcal{N}} \gamma_{\mu} \mathcal{N}
\end{aligned}
$$

one has

$$
g_{1 R}^{\mathrm{B}}=\frac{e^{2}}{\left(4 \pi \cos ^{2} \theta_{\mathrm{W}}\right)^{2}} V_{e \tau}^{\mathrm{e}} V_{\mu \tau}^{\mathrm{e} *} \frac{1}{72}\left[Z\left(32 c_{u_{R}}-2 c_{u_{L}}+4 c_{d_{R}}-c_{d_{L}}\right)+N\left(16 c_{u_{R}}-c_{u_{L}}+8 c_{d_{R}}-2 c_{d_{L}}\right)\right] .
$$

A numerical calculation shows that the contribution of the box diagrams to the decay rate goes significantly below the analogous contribution from the penguins as soon as $M_{2}$ moves away from zero, due to the rapid increase of the squark masses in the denominator and, even more so, to an increase of the magnetic contribution. In figure 13 we give the rate for $\mu \rightarrow e$ conversion in the $\mathrm{SU}(5)$ case and $\tan \beta=2$. The numerical results for $\tan \beta=10$ are not shown because they reproduce the relation (38) to a good approximation for any value of the others parameters.

\section{$11 \tau \rightarrow \mu \gamma$}

Very similar considerations to those developed in the previous sections can be made in the case of the $\tau \rightarrow \mu \gamma$ decay. A main point is the relative difference between the $\mathrm{SU}(5)$ and the $\mathrm{SO}(10)$ case.

In the $\mathrm{SU}(5)$ case, the amplitude for $\tau \rightarrow \mu \gamma$ is simply obtained from $\mathcal{A}_{\mu}(\mu \rightarrow e \gamma)$, equations $\left.18-19\right)$ with the replacement of the factor $m_{\mu} V_{\tau \mu}^{\mathrm{e} *} V_{\tau e}^{\mathrm{e}}$ by $m_{\tau} V_{\tau \tau}^{\mathrm{e} *} V_{\tau \mu}^{\mathrm{e}}$, up to negligible terms of relative order $m_{\mu} / m_{\tau}$ (remember that in $\mathrm{SU}(5) \tilde{\tau}_{L}$ and $\tilde{\mu}_{L}$ are degenerate to a very good accuracy). Consequently the following relation holds 3

$$
\left.\frac{\text { B.R. }(\tau \rightarrow \mu \gamma)}{\text { B.R. }(\mu \rightarrow e \gamma)}\right|_{\mathrm{SU}(5)}=\left|\frac{V_{\tau \tau}^{\mathrm{e}}}{V_{e \tau}^{\mathrm{e}}}\right|^{2} \text { B.R. }(\tau \rightarrow \mu \nu \bar{\nu}) \approx 3 \cdot 10^{3}\left(\frac{0.77}{y}\right)^{2}\left|\frac{0.01}{V_{t d}}\right|^{2}
$$

with $y$ given in (56). For given values of the mixing angles, this relation establishes the relative merit of the searches for the two decay processes as a possible signal of lepton flavour violation. With $\left|V_{t d}\right|=0.01$, the present limit on $\mu \rightarrow e \gamma\left(\right.$ B.R. $\left.<4.9 \cdot 10^{-11}\right)$ [16] is about 30 times better than the present bound on $\tau \rightarrow \mu \gamma\left(\right.$ B.R. $\left.<4.2 \cdot 10^{-6}\right)$ [18]. Using the relation (45), the contours of figures 7 and 8 can be relabelled with values of B.R. $(\tau \rightarrow \mu \gamma)$.

In $\mathrm{SO}(10)$, the $\mu \rightarrow e \gamma$ amplitude is proportional to $m_{\tau}$ and is therefore enhanced, as discussed in section 9. Consequently the ratio of the branching ratios will be further suppressed in SO(10), relative to eq. (45), by an approximate factor of order $\left(m_{\mu} / m_{\tau}\right)^{2}$.

\section{Electric dipole moment of the electron}

It has been pointed out by Dimopoulos and one of us (L.H.) [5] that, in an $\mathrm{SO}(10)$ unified theory, the low energy Lagrangian gives rise to an electric dipole moment for the neutron and the electron originating from the phases of the Yukawa couplings. We concentrate here on the dipole moment, $d_{e}$, of the electron, since in this case a very simple relation exists between $d_{e}$ and the $\mu \rightarrow e \gamma$ rate. It is clear however that the search for a dipole moment of the neutron constitutes an independent and equally important signature for the general effect discussed in this paper.

The full set of diagrams that contribute to the electric dipole or magnetic moments of the electron coincides with the one shown in fig. 10 with $\mu_{L}\left(\mu_{L}^{c}\right)$ replaced by $e_{L}\left(e_{L}^{c}\right)$. In particular, as readily seen from the different dependence on the CKM matrix elements, only the diagrams of figures $10 \mathrm{~b}, \mathrm{c}, \mathrm{c}^{\prime}$ contribute to the electric dipole moment (with $V_{\tau \mu}^{\mathrm{e}}$ replaced by $V_{\tau e}^{\mathrm{e}}$ ), since they are the only ones with an imaginary part. These are, on the other hand, the same diagrams that dominate the $\mu \rightarrow e \gamma$ amplitude through their

\footnotetext{
${ }^{3}$ This equation corrects eq. (21) of ref. [2].
} 
Figure 15: Same as in fig. 11 except for the scale of the initial condition on the RGEs taken at $2.0 \cdot 10^{17} \mathrm{GeV}$.

\begin{tabular}{l|rrc} 
GUT & $\tau \rightarrow \mu \gamma$ & $\mu \rightarrow e$ & $d_{e}$ \\
\hline $\mathrm{SU}(5)$ & 0.03 & $\gtrsim 0.2$ & 0 \\
$\mathrm{SO}(10)$ & $\ll 0.03$ & 0.2 & $2 \sin \varphi$
\end{tabular}

Table 1: Relative merits of various observables relative to $\mu \rightarrow e \gamma$. All branching ratios, as well as the value of $d_{e}$, are normalized to the present limits (B.R. $(\mu \rightarrow e \gamma)<4.9 \cdot 10^{-11}$ [16], C.R. $\left(\mu \rightarrow e\right.$ in Ti) $<10^{-12}$ [17], B.R. $\left.(\tau \rightarrow \mu \gamma)<4.2 \cdot 10^{-6}[18],\left|d_{e}\right|<4.3 \cdot 10^{-27} e \cdot \mathrm{cm}[19]\right)$.

$m_{\tau}$ dependent contribution. As a consequence, the following approximate relation holds between the form factor $F_{2}$ defined in eq. (31) and the electron dipole moment, $d_{e}$

$$
\left|d_{e}\right|=e\left|F_{2}\right|\left|\frac{V_{\tau e}^{\mathrm{e}}}{V_{\tau \mu}^{\mathrm{e}}}\right| \sin \varphi=e\left|F_{2}\right|\left|\frac{V_{t d}}{V_{t s}}\right| \sin \varphi
$$

with the $\mathrm{CP}$ violating phase $\varphi$ defined by

$$
\operatorname{Im}\left[m_{\tau}\left(V_{\tau e}^{\mathrm{e}}\right)^{2}\left(V_{\tau \tau}^{\mathrm{e} *}\right)^{2}\right] \equiv\left|m_{\tau}\left(V_{\tau e}^{\mathrm{e}}\right)^{2}\left(V_{\tau \tau}^{\mathrm{e} *}\right)^{2}\right| \sin \varphi .
$$

In $\mathrm{SO}(10)$ the electric dipole moment is therefore approximately related to the $\mu \rightarrow e \gamma$ branching ratio by

$$
\frac{\left|d_{e}\right|}{10^{-27} e \cdot \mathrm{cm}}=1.3 \sin \varphi \sqrt{\frac{\text { B.R. }(\mu \rightarrow e \gamma)}{10^{-12}}} .
$$

Using this relation, the contours of figures 11 and 12 can be relabelled with values of $\left|d_{e}\right| / \sin \varphi$. It is interesting to notice that the present upper bound on $\mu \rightarrow e \gamma\left(\right.$ B.R. $\left.<4.9 \cdot 10^{-11}\right)$ and $d_{e}\left(\left|d_{e}\right|<\right.$ $4.3 \cdot 10^{-27} e \cdot \mathrm{cm}$ ] [19] are almost exactly equivalent for $\sin \varphi=1 / 2$.

As in $\mathrm{SO}(10)$, in $\mathrm{SU}(5)$ too, the diagrams that could contribute to the electric dipole moment of the electron are obtained from those of figure 6 by replacing the muon with the electron in the external line. This time, however, no electric dipole moment arises since the $\mathrm{CP}$ violating phase disappears from the product $V_{\tau e}^{\mathrm{e} *} V_{\tau e}^{\mathrm{e}}$ of the relevant CKM matrix elements.

\section{Conclusions}

In this paper we have discussed the lepton flavour violating processes and electric dipole moments induced in a supersymmetric unified theory by the large top Yukawa coupling. Under the stated assumptions, the experimental study of these processes provides a very significant test of supersymmetric unification. Already the present experimental limits give, especially in the $\mathrm{SO}(10)$ case, significant restrictions on the allowed parameter space, often considerably stronger than those inferred from direct searches of supersymmetric particles.

The main results of this paper are the contour plots for B.R. $(\mu \rightarrow e \gamma)$ shown in figures 7 and 8 , for $\mathrm{SU}(5)$, and in figures 11 and 12 , for $\mathrm{SO}(10)$. The figures 7 and 8 can also be used for $\tau \rightarrow \mu \gamma$ by a relabelling of the contours using equation (45). Similarly the contours of figures 11 and 12 can be relabelled using (38) and (47) so that they apply to $\mu \rightarrow e$ conversion and to $d_{e}$ respectively. The case of $\mu \rightarrow e$ conversion in $\mathrm{SU}(5)$ is shown in figure 13 . These plots cover the entire physical ranges of the parameters $A_{e}$ and $M_{2}$, and show the behaviour for both signs of $\mu$ and for both large and small values of $\tan \beta$. For large $m_{\tilde{e}_{R}}$ the B.R. decreases as $1 / m_{\tilde{e}_{R}}^{4}$; however values of $m_{\tilde{e}_{R}}$ above $400 \mathrm{GeV}$ require a significant amount of fine tuning [20].

The sensitivities of the various processes to the $\mathrm{SU}(5)$ and $\mathrm{SO}(10)$ theories is summarized in table 1 , relative to that of $\mu \rightarrow e \gamma$, with all observables normalized to the present experimental bounds. For $\sin \varphi<1 / 2$ all entries of this table are less than unity, showing that, for this case, $\mu \rightarrow e \gamma$ is presently the most powerful probe in all cases. For $\sin \varphi>1 / 2$ the electron electric dipole moment provides the best probe of the $\mathrm{SO}(10)$ theory. The decay $\tau \rightarrow \mu \gamma$ will only become competitive with the construction of a 
$\tau$ factory. Future technologies and experimental possibilities should allow an interesting competition to develop amongst the other three processes.

An additional prediction of this work is that the mass of $\tilde{\tau}_{R}$ is suppressed significantly beneath that of $\tilde{\mu}_{R}$ and $\tilde{e}_{R}$, as can be seen in figure 1 . In addition, in $\mathrm{SO}(10)$ the mass of $\tilde{\tau}_{L}$ is suppressed beneath that of $\tilde{\mu}_{L}$ and $\tilde{e}_{L}$. This result is important for superpartner searches at $e^{+} e^{-}$colliders: the lightest charged scalar superpartner is almost certainly a scalar tau.

The sizes of these effects depend on the following two main assumptions:

i) The value of the top Yukawa coupling at the unification scale is large;

ii) The field theoretic renormalization group equations, valid at the unification scale, can be extrapolated without substantial modifications up to the reduced Planck mass, $M_{\mathrm{Pl}}=2.4 \cdot 10^{17} \mathrm{GeV}$, or to a scale close to it, e.g. the compactification scale of string theory.

A value of the top Yukawa coupling at the unification scale less than one leads to a substantial reduction of the rates, as shown, e.g., in fig. 12 as compared to fig. 11. Also significant, although relatively less important, is the lowering of the scale for the universal conditions on the supersymmetry breaking parameters, as exemplified in figure 15 where such scale is taken at $2.0 \cdot 10^{17} \mathrm{GeV}$.

What other features of the unified model influence our results? Other than on the gauge group itself, the lepton flavour violation effects certainly depend on the specific form of the flavour interactions. In this paper we have studied the two simplest unified sectors that we know, one in SU(5) and the other in $\mathrm{SO}(10)$. Each has the minimal number of flavour Yukawa matrices, $\boldsymbol{\lambda}^{\mathrm{u}}$ giving mass to up quarks and $\lambda^{\mathrm{d}}$ to down quarks and charged leptons. It is well known that these Yukawa couplings lead to unrealistic mass relations between the light fermions. However, as already discussed in ref. [2], we do not expect that the necessary modifications of these couplings may lead to significant suppressions of the lepton flavour violation processes. They will rather give rise to an increased range of predictions about the central values discussed here.

The traditional probes of supersymmetric unified theories are provided by proton decay, neutrino masses and by predictions for quark and charged lepton masses and mixings. These probes also have model dependences which arise from the choice of the gauge group and the flavour interactions, as discussed above. However, for each of these three probes, there is also a much greater uncertainty than in the lepton flavour violation processes. A generic unified model has a free parameter for each of the flavour masses and mixing parameters of the standard model, and hence does not make predictions for the quark and charged lepton masses. Such predictions only arise when the form of the flavour interactions are restricted by further assumptions.

While proton decay and neutrino masses are generally to be expected in superunified models, the sizes of these signatures are extremely model dependent. Consider first the case of proton decay. All superunified models contain baryon and lepton number violating interactions which couple the quarks and leptons to a set of superheavy coloured states $H$. The amplitude for proton decay depends on the mass matrix for these $H$ states. This is perhaps the least understood, and most model dependent, feature of superunified theories, because it is directly related to the problem of why the Higgs doublets are much lighter than $M_{\mathrm{G}}$. Only in one particular model [6], where the Higgs are made light by an extreme fine tune, has it been possible to relate the $H$ mass to known parameters of the theory and hence make predictions for the proton decay rate. In fact the resulting rate is large, and this model is close to being excluded. In many other models the matrix structure of the masses for the $H$ states leads to a large suppression of the proton decay amplitude, which then becomes gauge dominated, yielding a rate which is expected to be about four orders of magnitude below present experimental limits.

The three neutrinos frequently acquire small masses in superunified models, particularly if the gauge group contains $\mathrm{SO}(10)$. However, the size of these masses is inversely proportional to $\boldsymbol{M}_{R}$, the Majorana mass matrix for the right-handed neutrinos, which breaks lepton number and is typically not directly related to known parameters of the theory. A simple expectation of $M_{R} \approx M_{\mathrm{G}}$ gives masses for $\nu_{e}, \nu_{\mu}, \nu_{\tau}$ which are too small to see in accelerator or reactor experiments.

By comparison with these great uncertainties, which afflict the traditional signatures for superunified models, the model dependence of the rates for $L_{e}, L_{\mu}, L_{\tau}$ and CP violating processes discussed here seems quite mild.

We therefore conclude that searches for the $L_{i}$ and $\mathrm{CP}$ violating signatures discussed in this paper provide the most powerful known probes of supersymmetric quark-lepton unification with supersymmetry 


$$
\begin{array}{llll}
\mathrm{SU}(5) & b_{g}^{\mathrm{u}}=\{3,3,9\} & c^{\mathrm{u}}=96 / 5 & c^{H}=24 / 5 \\
& b_{g}^{\mathrm{d}}=\{0,0,3\} & c^{\mathrm{d}}=84 / 5 & c^{T}=36 / 5 \\
\mathrm{SO}(10) & b_{g}^{\mathrm{u}}=\{4,4,14\} & c^{\mathrm{u}}=63 / 2 & c^{\Phi}=9 \\
& b_{g}^{\mathrm{d}}=\{0,0,10\} & c^{\mathrm{d}}=63 / 2 & c^{\Psi}=45 / 4
\end{array}
$$

Table 2: Values of the RGE coefficients in SU(5) and in $\mathrm{SO}(10)$.

breaking generated at the Planck scale. For example, an experiment with a sensitivity of $10^{-13}$ to B.R. $(\mu \rightarrow$ $e \gamma$ ) would probe (apart from a small region of parameter space where cancellations in the amplitude occur) the SU(5) model to $\lambda_{t \mathrm{G}}=1.4$ and $m_{\tilde{e}_{R}}=100 \mathrm{GeV}$, and would explore a significant portion of parameters space for $m_{\tilde{e}_{R}}=300 \mathrm{GeV}$. In the $\mathrm{SO}(10)$ case, where the present bound on $\mu \rightarrow e \gamma$ is already more stringent than the limits from high energy accelerator experiments, a sensitivity of $10^{-13}$ would probe the theory to $\lambda_{t \mathrm{G}}=1.25$ and $m_{\tilde{e}_{R}}$ close to $1 \mathrm{TeV}$.

\section{A Renormalization from the Planck to the GUT scale}

Neglecting all couplings except the gauge and the top Yukawa ones, the solutions to all the one loop RGEs between $E_{\max }=M_{\mathrm{Pl}}$ and $E_{\min }=M_{\mathrm{G}}$ can be given analytically.

The RGEs for the dimensionless couplings and for the dimension-one soft terms are

$$
\begin{aligned}
\frac{d}{d t} \frac{1}{\alpha_{5}} & =4 \pi b_{\mathrm{G}} \quad \frac{d}{d t} \frac{M_{5}}{\alpha_{5}}=0 \\
\frac{d}{d t} \lambda_{t}^{2} & =\lambda_{t}^{2}\left(c^{\mathrm{u}} g_{5}^{2}-b_{t} \lambda_{t}^{2}\right) \\
\frac{d}{d t} A_{g}^{\mathrm{u}} & =c^{\mathrm{u}} g_{5}^{2} M_{5}-b_{g}^{\mathrm{u}} \lambda_{t}^{2} A_{t} \\
\frac{d}{d t} A_{g}^{\mathrm{d}} & =c^{\mathrm{d}} g_{5}^{2} M_{5}-b_{g}^{\mathrm{d}} \lambda_{t}^{2} A_{t}
\end{aligned}
$$

where $t(E)=(4 \pi)^{-2} \ln M_{\mathrm{Pl}}^{2} / E^{2}, g_{5}$ is the coupling constant of the unification group, $\alpha_{5}=g_{5}^{2} / 4 \pi, M_{5}$ is the gaugino mass, $g=1,2,3$ is the generation number and the values of the numerical coefficients in $\mathrm{SU}(5)$ and in $\mathrm{SO}(10)$ are given in table 2. The subscript ' 5 ' stands for 'unified' rather than for $\mathrm{SU}(5)$. We also set $b_{3}^{\mathrm{u}} \equiv b_{t}$.

The full analytic solutions of these equations with the boundary conditions

$$
\alpha_{5}\left(M_{\mathrm{G}}\right)=\alpha_{\mathrm{G}}, \quad M_{5}\left(M_{\mathrm{G}}\right)=M_{5 \mathrm{G}}, \quad \lambda_{t}\left(M_{\mathrm{G}}\right)=\lambda_{t \mathrm{G}}
$$

and universal $A$-terms at the Planck scale, $A_{0}$, are

$$
\begin{aligned}
\alpha_{5}(E) & =f_{5}^{-1}(E) \cdot \alpha_{\mathrm{G}} \\
M_{5}(E) & =f_{5}^{-1}(E) \cdot M_{5 \mathrm{G}} \\
\lambda_{t}^{2}(E) & =\frac{\lambda_{t}^{2 \max }(E)}{1+\lambda_{t}^{2 \max }(E)\left(\lambda_{t \mathrm{G}}^{-2}-\lambda_{t \mathrm{G}}^{-2 \mathrm{max}}\right) f_{5}^{-c^{\mathrm{u}} / b_{\mathrm{G}}}(E)} \\
A_{g}^{\mathrm{u}}(E) & =A_{0}+x_{1}^{\mathrm{u}}(E) M_{5 \mathrm{G}}-b_{g}^{\mathrm{u}} I^{\prime}(E) / b_{t} \\
A_{g}^{\mathrm{d}}(E) & =A_{0}+x_{1}^{\mathrm{d}}(E) M_{5 \mathrm{G}}-b_{g}^{\mathrm{d}} I^{\prime}(E) / b_{t}
\end{aligned}
$$

where the functions $f_{5}(E), x_{n}^{R}(E), \lambda_{t}^{\max }(E), I^{\prime}(E)$ are explicitly defined below in eq. (50).

Assuming universal values at the Planck scale for the dimension-two supersymmetry breaking soft terms, $m_{R}^{2}=m_{0}^{2}$, the one loop RGEs in $\mathrm{SU}(5)$

$$
\begin{aligned}
\frac{d}{d t} m_{\bar{F}_{g}}^{2} & =2 c^{H} g_{5}^{2} M_{5}^{2} \\
\frac{d}{d t} m_{H}^{2} & =2 c^{H} g_{5}^{2} M_{5}^{2}-\frac{b_{t}}{3} \lambda_{t}^{2}\left(2 m_{T_{3}}^{2}+m_{H}^{2}+A_{t}^{2}\right)
\end{aligned}
$$


are solved by

$$
\frac{d}{d t} m_{T_{g}}^{2}=2 c^{T} g_{5}^{2} M_{5}^{2}-\frac{b_{t}}{3} \lambda_{t}^{2}\left(2 m_{T_{3}}^{2}+m_{H}^{2}+A_{t}^{2}\right)
$$

$$
\begin{aligned}
m_{\bar{F}_{g}}^{2}(E) & =m_{0}^{2}+x_{2}^{H}(E) M_{5 \mathrm{G}}^{2} \\
m_{H}^{2}(E) & =m_{0}^{2}+x_{2}^{H}(E) M_{5 \mathrm{G}}^{2}-I(E) \\
m_{T_{g}}^{2}(E) & =m_{0}^{2}+x_{2}^{T}(E) M_{5 \mathrm{G}}^{2}-I(E) \delta_{g 3}
\end{aligned}
$$

and in $\mathrm{SO}(10)$

by

$$
\begin{aligned}
\frac{d}{d t} m_{\Phi}^{2} & =2 c^{\Phi} g_{5}^{2} M_{5}^{2}-\frac{4}{14} b_{t} \lambda_{t}^{2}\left(2 m_{\Psi_{3}}^{2}+m_{\Phi}^{2}+A_{t}^{2}\right) \\
\frac{d}{d t} m_{\Psi_{g}}^{2} & =2 c^{\Psi} g_{5}^{2} M_{5}^{2}-\frac{5}{14} b_{t} \lambda_{t}^{2}\left(2 m_{\Psi_{3}}^{2}+m_{\Phi}^{2}+A_{t}^{2}\right)
\end{aligned}
$$

$$
\begin{aligned}
m_{\Phi}^{2}(E) & =m_{0}^{2}+x_{2}^{\Phi}(E) M_{5 \mathrm{G}}^{2}-3 \frac{4}{14} I(E) \\
m_{\Psi_{g}}^{2}(E) & =m_{0}^{2}+x_{2}^{\Psi}(E) M_{5 \mathrm{G}}^{2}-3 \frac{5}{14} I(E) \delta_{g 3}
\end{aligned}
$$

In both cases, we have defined

$$
\begin{aligned}
f_{5}(E) \equiv & 1+g_{\mathrm{G}}^{2} b_{\mathrm{G}}\left[t(E)-t\left(M_{\mathrm{G}}\right)\right] \\
x_{n}^{R}(E) \equiv & \frac{c^{R}}{b_{\mathrm{G}}}\left[f_{5}^{-n}\left(E_{\max }\right)-f_{5}^{-n}(E)\right] \\
\lambda_{t}^{2 \max }(E) \equiv & \frac{c^{\mathrm{u}}+b_{\mathrm{G}}}{b_{t}} \frac{g_{5}^{2}(E)}{1-\left[f_{5}\left(E_{\max }\right) / f_{5}(E)\right]^{1+c^{\mathrm{u}} / b_{\mathrm{G}}}} \\
I(E) \equiv & \rho\left[m_{0}^{2}+\frac{1}{3}(1-\rho) A_{0}^{2}-\frac{2}{3}(1-\rho)\left(1-b_{t} \lambda_{t}^{2 \max } t\right) A_{0} M_{5 \mathrm{Pl}}\right. \\
& \left.-\frac{1}{3}\left[\rho\left(1-b_{t} \lambda_{t}^{2 \max } t\right)^{2}-b_{t} c^{\mathrm{u}} \lambda_{t}^{2 \max } g_{5 \mathrm{G}}^{2} t^{2}\right] M_{5 \mathrm{Pl}}^{2}\right] \\
I^{\prime}(E) \equiv & \rho\left[A_{0}-\left(1-b_{t} \lambda_{t}^{2 \max } t\right) M_{5 \mathrm{Pl}}\right]
\end{aligned}
$$

and $\rho(E) \equiv \lambda_{t}^{2}(E) / \lambda_{t}^{2 \max }(E)<1$. From eq.s $(50)$, one learns that the main factor that determines the size of the lepton flavour breaking parameters $I$ and $I^{\prime}$ is the overall factor $\rho$. In turn, $\rho$ is only weakly dependent on the $\beta$-function coefficient $b_{\mathrm{G}}$ (see figure 3).

For the numerical values at $M_{\mathrm{G}}$ of the different quantities defined above we take

$$
\alpha_{\mathrm{G}}=1 / 24, \quad M_{\mathrm{G}}=2.0 \cdot 10^{16} \mathrm{GeV}, \quad M_{\mathrm{Pl}}=2.4 \cdot 10^{18} \mathrm{GeV}, \quad b_{\mathrm{G}}=-3,
$$

so that $t_{\mathrm{G}}=0.0606$ (a subscript ' $\mathrm{G}$ ' on the various functions of $E$ indicates that they are evaluated at $\left.M_{\mathrm{G}}\right)$. In $\mathrm{SU}(5) \lambda_{t \mathrm{G}}^{\max }=1.56$ and

$$
\begin{aligned}
I_{\mathrm{G}} \equiv I\left(M_{\mathrm{G}}\right) & =\rho_{\mathrm{G}}\left[m_{0}^{2}+\frac{1}{3}\left(1-\rho_{\mathrm{G}}\right) A_{0}^{2}+0.198\left(1-\rho_{\mathrm{G}}\right) A_{0} M_{5 \mathrm{G}}+\left(0.224-0.029 \rho_{\mathrm{G}}\right) M_{5 \mathrm{G}}^{2}\right] \\
I_{\mathrm{G}}^{\prime} \equiv I^{\prime}\left(M_{\mathrm{G}}\right) & =\rho_{\mathrm{G}}\left[A_{0}+0.298 M_{5 \mathrm{G}}\right]
\end{aligned}
$$

while in $\mathrm{SO}(10) \lambda_{t \mathrm{G}}^{\max }=1.36$ and

$$
\begin{aligned}
I_{\mathrm{G}} \equiv I\left(M_{\mathrm{G}}\right) & =\rho_{\mathrm{G}}\left[m_{0}^{2}+\frac{1}{3}\left(1-\rho_{\mathrm{G}}\right) A_{0}^{2}+0.343\left(1-\rho_{\mathrm{G}}\right) A_{0} M_{5 \mathrm{G}}+\left(0.435-0.088 \rho_{\mathrm{G}}\right) M_{5 \mathrm{G}}^{2}\right] \\
I_{\mathrm{G}}^{\prime} \equiv I^{\prime}\left(M_{\mathrm{G}}\right) & =\rho_{\mathrm{G}}\left[A_{0}+0.515 M_{5 \mathrm{G}}\right]
\end{aligned}
$$

\section{B Renormalization in the MSSM}

Neglecting all couplings except the gauge and the top Yukawa ones, the solutions to all the one loop RGEs between $E_{\max }=M_{\mathrm{G}}$ and $E_{\min }=M_{Z}$ may be written in terms of analytic functions and only one function, $\lambda_{t}^{\max }(E)$, calculable only numerically [21]. 


\begin{tabular}{r|ccccc|ccc||c||ccc}
$b_{i}$ & $c_{i}^{Q}$ & $c_{i}^{u}$ & $c_{i}^{d}$ & $c_{i}^{L}$ & $c_{i}^{e}$ & $c_{i}^{\mathrm{u}}$ & $c_{i}^{\mathrm{d}}$ & $c_{i}^{\mathrm{e}}$ & $i, g$ & $b_{g}^{\mathrm{u}}$ & $b_{g}^{\mathrm{d}}$ & $b_{g}^{\mathrm{e}}$ \\
\hline$\frac{33}{5}$ & $\frac{1}{30}$ & $\frac{8}{15}$ & $\frac{2}{15}$ & $\frac{3}{10}$ & $\frac{6}{5}$ & $\frac{13}{15}$ & $\frac{7}{15}$ & $\frac{9}{5}$ & 1 & 3 & 0 & 0 \\
1 & $\frac{3}{2}$ & 0 & 0 & $\frac{3}{2}$ & 0 & 3 & 3 & 3 & 2 & 3 & 0 & 0 \\
-3 & $\frac{8}{3}$ & $\frac{8}{3}$ & $\frac{8}{3}$ & 0 & 0 & $\frac{16}{3}$ & $\frac{16}{3}$ & 0 & 3 & 6 & 1 & 0
\end{tabular}

Table 3: Values of the RGE coefficients in the MSSM.

The RGEs for the dimensionless couplings are

$$
\begin{aligned}
\frac{d}{d t} \frac{1}{\alpha_{i}} & =4 \pi b_{i} \\
\frac{d}{d t} \lambda_{t}^{2} & =\lambda_{t}^{2}\left(c_{i}^{\mathrm{u}} g_{i}^{2}-b_{t} \lambda_{t}^{2}\right)
\end{aligned}
$$

where $i=1,2,3$ runs over the three factor in the Standard Model gauge group $\mathrm{U}(1) \otimes \mathrm{SU}(2) \otimes \mathrm{SU}(3)$, $t(E)=(4 \pi)^{-2} \ln M_{\mathrm{G}}^{2} / E^{2}$ and the values of the coefficients are shown in table 3 .

The solutions with boundary conditions $\alpha_{i}\left(M_{\mathrm{G}}\right)=\alpha_{\mathrm{G}}$ and $\lambda_{t}\left(M_{\mathrm{G}}\right)=\lambda_{t \mathrm{G}}$ are

$$
\begin{aligned}
\alpha_{i}(E) & =f_{i}^{-1}(E) \cdot \alpha_{\mathrm{G}} \\
\lambda_{t}^{2}(E) & =\frac{\lambda_{t}^{2 \max }(E)}{1+\lambda_{t}^{2 \max }(E) / \lambda_{t \mathrm{G}}^{2} E_{\mathrm{u}}(E)}
\end{aligned}
$$

where $f_{i}(E) \equiv 1+b_{i} g_{\mathrm{G}}^{2} t(E)$ and

$$
E_{\alpha}(E) \equiv \prod_{i} f_{i}^{c_{i}^{\alpha} / b_{i}}(E), \quad F_{\mathrm{u}}(E) \equiv 2 \int_{\ln E}^{\ln M_{\mathrm{G}}} E_{\mathrm{u}}(E) d \ln E, \quad \lambda_{t}^{2 \max }(E)=\frac{E_{\mathrm{u}}(E)}{b_{t} F_{\mathrm{u}}(E)}
$$

The Yukawa couplings of the fermions in the diagonal basis scale as $\lambda_{g}^{\alpha}(E)=\lambda_{g}^{\alpha}\left(M_{\mathrm{G}}\right) \cdot y^{b_{g}^{\alpha}} E_{\alpha}^{1 / 2}$ where $g=1,2,3$ is the generation number, $\alpha=\mathrm{u}, \mathrm{d}$, e and

$$
y(E) \equiv \exp \left[-\int_{\ln E}^{\ln M_{\mathrm{G}}} \frac{\lambda_{t}^{2}\left(E^{\prime}\right)}{16 \pi^{2}} d \ln E^{\prime}\right]=[1-\rho(E)]^{1 / 2 b_{t}}, \quad \rho(E) \equiv \frac{\lambda_{t}^{2}(E)}{\lambda_{t}^{2 \max }(E)}<1 .
$$

The factor $y$ used in the text is given by $y \equiv y\left(M_{Z}\right)$.

The RGEs for the three gaugino masses $M_{i}$, the supersymmetric $\mu$-term and the $A$ terms are

$$
\begin{aligned}
\frac{d}{d t} \frac{M_{i}}{\alpha_{i}} & =0 \\
\frac{d}{d t} \mu & =\frac{1}{2}\left(2 c_{i}^{h} g_{i}^{2}-b_{1}^{\mathrm{u}} \lambda_{t}^{2}\right) \mu \\
\frac{d}{d t} A_{u, g} & =c_{i}^{\mathrm{u}} g_{i}^{2} M_{i}-b_{g}^{\mathrm{u}} \lambda_{t}^{2} A_{u, g} \\
\frac{d}{d t} A_{d, g} & =c_{i}^{\mathrm{d}} g_{i}^{2} M_{i}-b_{g}^{\mathrm{d}} \lambda_{t}^{2} A_{d, g} \\
\frac{d}{d t} A_{e, g} & =c_{i}^{\mathrm{e}} g_{i}^{2} M_{i}
\end{aligned}
$$

with all the various coefficients listed in table 3. The solutions are

$$
\begin{aligned}
M_{i}(E) & =f_{i}^{-1}(E) \cdot M_{5 \mathrm{G}} \\
\mu(E) & =\mu\left(M_{5 \mathrm{G}}\right) \cdot y^{b_{1}^{\mathrm{u}}}(E) E_{h}(E) \\
A_{g}^{\mathrm{u}}(E) & =A_{g \mathrm{G}}^{\mathrm{u}}+x_{1}^{\mathrm{u}}(E) M_{5 \mathrm{G}}-b_{g}^{\mathrm{u}} I^{\prime}(E) / b_{t} \\
A_{g}^{\mathrm{d}}(E) & =A_{g \mathrm{G}}^{\mathrm{d}}+x_{1}^{\mathrm{d}}(E) M_{5 \mathrm{G}}-b_{g}^{\mathrm{d}} I^{\prime}(E) / b_{t} \\
A_{g}^{\mathrm{e}}(E) & =A_{g \mathrm{G}}^{\mathrm{e}}+x_{1}^{\mathrm{e}}(E) M_{5 \mathrm{G}}
\end{aligned}
$$


with $x_{1}^{R}(E)$ and $I^{\prime}(E)$ defined below in (60) and $E_{h}(E)$ in (55).

The RGEs for the dimension-two soft parameters of a representation $R=\{Q, u, d, e, L=h\}$ are

$$
\frac{d}{d t} m_{R}^{2}=2 c_{i}^{R} g_{i}^{2} M_{i}^{2}
$$

except for the multiplets $h_{\mathrm{u}}, \tilde{Q}_{3}$ and $\tilde{t}$ involved in the top Yukawa coupling. For them

$$
\begin{aligned}
\frac{d}{d t} m_{h_{\mathrm{u}}}^{2} & =2 c_{i}^{h} g_{i}^{2} M_{i}^{2}-\frac{1}{2} b_{t} \lambda_{t}^{2}\left(A_{t}^{2}+3 m^{2}\right) \\
\frac{d}{d t} m_{\tilde{Q}_{3}}^{2} & =2 c_{i}^{Q} g_{i}^{2} M_{i}^{2}-\frac{1}{6} b_{t} \lambda_{t}^{2}\left(A_{t}^{2}+3 m^{2}\right) \\
\frac{d}{d t} m_{\tilde{t}}^{2} & =2 c_{i}^{u} g_{i}^{2} M_{i}^{2}-\frac{1}{3} b_{t} \lambda_{t}^{2}\left(A_{t}^{2}+3 m^{2}\right)
\end{aligned}
$$

where $m^{2}(E) \equiv\left[m_{h_{\mathrm{u}}}^{2}(E)+m_{\tilde{Q}_{3}}^{2}(E)+m_{\tilde{t}}^{2}(E)\right] / 3$. The solutions are

$$
m_{R}^{2}(E)=m_{R}^{2}\left(M_{\mathrm{G}}\right)+x_{2}^{R} M_{5 \mathrm{G}}^{2}
$$

except when the top Yukawa coupling appears, where

$$
\begin{aligned}
m_{h_{\mathrm{u}}}^{2}(E) & =m_{h_{\mathrm{u}}}^{2}\left(M_{\mathrm{G}}\right)+x_{2}^{h}(E) M_{5 \mathrm{G}}^{2}-\frac{1}{2} 3 I(E) \\
m_{\tilde{Q}_{3}}^{2}(E) & =m_{10_{3}}^{2}\left(M_{\mathrm{G}}\right)+x_{2}^{Q}(E) M_{5 \mathrm{G}}^{2}-\frac{1}{6} 3 I(E) \\
m_{\tilde{t}}^{2}(E) & =m_{10_{3}}^{2}\left(M_{\mathrm{G}}\right)+x_{2}^{u}(E) M_{5 \mathrm{G}}^{2}-\frac{1}{3} 3 I(E)
\end{aligned}
$$

and

$$
\begin{aligned}
x_{n}^{R}(E) \equiv & \sum_{i=1}^{3} \frac{c_{i}^{R}}{b_{i}}\left[f_{i}^{-n}\left(E_{\max }\right)-f_{i}^{-n}(E)\right] \\
I(E) \equiv & \rho\left[m^{2}\left(M_{\mathrm{G}}\right)+\frac{1}{3}(1-\rho) A_{t \mathrm{G}}^{2}-\frac{2}{3}(1-\rho)\left(1-b_{t} \lambda_{t}^{2 \max } t\right) M_{5 \mathrm{G}} A_{t \mathrm{G}}-\right. \\
& \left.\frac{1}{3}\left[\rho\left(1-b_{t} \lambda_{t}^{2 \max } t\right)^{2}-b_{t} \lambda_{t}^{2 \max } t^{2}\left(c_{i}^{\mathrm{u}} g_{i}^{2}\right)\right] M_{5 \mathrm{G}}^{2}\right] \\
I^{\prime}(E) \equiv & \rho\left[A_{t \mathrm{G}}-\left(1-b_{t} \lambda_{t}^{2 \max } t\right) M_{5 \mathrm{G}}\right]
\end{aligned}
$$

Notice that, apart form obvious replacements, $x_{n}^{R}, I$ and $I^{\prime}$ maintain exactly the same form as in eq. (50).

The numerical values at $M_{Z}$ of the different quantities defined above are $t\left(M_{Z}\right)=t_{Z}=0.418$ (a subscript ' $Z$ ' on the various functions of $E$ indicates that they are evaluated at $M_{Z}$ ),

$$
\begin{aligned}
& E_{\mathrm{u} Z}=13.6, \quad b_{t} F_{\mathrm{u} Z}=10.5, \quad \lambda_{t Z}^{\max }=1.14 \\
& I_{Z}=\rho_{Z} m\left(M_{\mathrm{G}}^{2}\right)+\frac{\rho_{Z}}{3}\left(1-\rho_{Z}\right) A_{t \mathrm{G}}^{2}+1.50 \rho_{Z}\left(1-\rho_{Z}\right) M_{5 \mathrm{G}} A_{t \mathrm{G}}+\rho_{Z}\left(4.37-1.70 \rho_{Z}\right) M_{5 \mathrm{G}}^{2} \\
& I_{Z}^{\prime}=\rho_{Z}\left[A_{t \mathrm{G}}+2.26 M_{5 \mathrm{G}}\right] \\
& f_{1 Z}=2.44 \quad f_{2 Z}=1.22 \quad f_{3 Z}=0.343 \\
& \begin{array}{lllll} 
& x_{1 Z}^{\mathrm{u}}=4.02 & x_{1 Z}^{\mathrm{d}}=3.98 & x_{1 Z}^{\mathrm{e}}=0.700 \\
x_{2 Z}^{Q}=7.16 & x_{2 Z}^{u}=6.73 & x_{2 Z}^{d}=6.68 & x_{2 Z}^{e}=0.151 \quad x_{2 Z}^{L}=x_{2 Z}^{h}=0.528
\end{array}
\end{aligned}
$$

Finally the Higgs doublets mass parameters $m_{\mathrm{d}}^{2} \equiv m_{h_{\mathrm{d}}}^{2}\left(M_{Z}\right)$ and $m_{\mathrm{u}}^{2} \equiv m_{h_{\mathrm{u}}}^{2}\left(M_{Z}\right)$ defined in eq. 10 may be expressed in terms of the universal supersymmetry breaking parameters as

in $\mathrm{SU}(5)$, while, in $\mathrm{SO}(10)$

$$
m_{\mathrm{d}}^{2}=m_{0}^{2}+\left(x_{2 \mathrm{G}}^{H}+x_{2 Z}^{h}\right) M_{5 \mathrm{G}}^{2}, \quad m_{\mathrm{u}}^{2}=m_{\mathrm{d}}^{2}-\left(\frac{3}{2} I_{Z}+I_{\mathrm{G}}\right),
$$

$$
m_{\mathrm{d}}^{2}=m_{0}^{2}+\left(x_{2 \mathrm{G}}^{\Phi}+x_{2 Z}^{h}\right) M_{5 \mathrm{G}}^{2}, \quad m_{\mathrm{u}}^{2}=m_{\mathrm{d}}^{2}-\left(\frac{3}{2} I_{Z}+\frac{6}{7} I_{\mathrm{G}}\right) .
$$




\section{References}

[1] L.J. Hall, V. A. Kostelecky and S. Raby, Nucl. Phys. B267 (1986) 415.

[2] R. Barbieri and L.J. Hall, Phys. Lett. B338 (1994) 212.

[3] H. Georgi and S. Glashow, Phys. Rev. Lett. 32 (1974) 438.

[4] H. Georgi in 'Particles and Fields', Proceedings of the APS Div. of Particles and Fields, ed. C. Carlson; H. Fritzsch and P. Minkowski, Ann. Phys. 93 (1975) 193.

[5] S. Dimopoulos and L.J. Hall, LBL 36269 (1994). To appear in Phys. Lett. B.

[6] S. Dimopoulos and H. Georgi, Nucl. Phys. B193 (1981) 150.

[7] R. Barbieri, S. Ferrara and C. Savoy, Phys. Lett. B110 (1982) 343;

P. Nath, R. Arnowitt and A. Chamseddine, Phys. Rev. Lett. 49 (1982) 970;

L.J. Hall, J. Lykken and S. Weinberg, Phys. Rev. D27 (1983) 2359.

[8] H. Arason et al., Phys. Rev. D46 (1992) 3945.

[9] M. Chanowitz, J. Ellis, and M.K. Gaillard, Nucl. Phys. B128 (1977) 506;

A. Buras, J. Ellis, M.K. Gaillard, and D.V. Nanopoulos, Nucl. Phys. B135 (1978) 66.

[10] L.E. Ibañez and C. Lopez, Phys. Lett. B126 (1983) 54; Nucl. Phys. B233 (1984) 511;

H. Arason et al., Phys. Rev. Lett. 67 (1991) 2933;

A. Giveon, L.J. Hall and U. Sarid, Phys. Lett. B271 (1991) 138;

S. Kelley, J.L. Lopez and D.V. Nanopoulos, Phys. Lett. B274 (1992) 387.

[11] CDF Coll., F. Abe et al., FermiLab-PUB-94/097-E (1994).

[12] M. Olechowski and S. Pokorski, Phys. Lett. B257 (1991) 388.

[13] F. Borzumati and A. Masiero, Phys. Rev. Lett. 57 (1986) 261;

F. Gabbiani and A. Masiero, Phys. Lett. B209 (1988) 289;

J.S. Hagelin, S. Kelley and T. Tanaka, Nucl. Phys. B415 (1994) 293.

[14] J. Bernabéu, E. Nardi, and D. Tommasini, Nucl. Phys. B409 (1993) 69, and references therein.

[15] T. Suzuki, D. Measday and J. Roalsvig, Phys. Rev. D46 (1992) 3040.

[16] R. Bolton et al., Phys. Rev. D38 (1988) 2077.

[17] Sindrum Collaboration, as quoted by R. Patterson, talk given at the International Conference on High Energy Physics, Glasgow, July 1994.

[18] A. Bean et al., Phys. Rev. Lett. 70 (1993) 138.

[19] E.D. Commins, S.B. Ross, D. Demille and B.C. Regan, Phys. Rev. A50 (1994) 2960.

[20] R. Barbieri and G.F. Giudice, Nucl. Phys. B306 (1988) 63.

[21] A. Bouquet, J. Kaplan and C.A. Savoy, Nucl. Phys. B262 (1985) 299. 